\title{
Synthesis of 6-oxopyrimidin-1(6H)-yl benzamide derivatives and evaluation of their antibacterial and cytotoxic activity
}

\author{
Kiran Devarasetty, ${ }^{\text {a,b* }}$ Giri Tharikoppula, ${ }^{\text {a,b }}$ Tailor Sridhar, ${ }^{a}$ Laxminarayana \\ Eppakayala, ${ }^{\mathrm{c}}$ Mahesh Kyasani, ${ }^{\mathrm{a}}$ Premkumar Arumugam ${ }^{\mathrm{a}}$ and Srinivas \\ Pusuluri $^{\text {a }}$ \\ ${ }^{a}$ Chemistry Services Unit, GVK biosciences Pvt. Ltd, Plot No. 5C, IDA, Uppal, Hyderabad \\ 500039, AP, India \\ (Phone: +91-7075675772; Email: devarasettykiran@gvkbio.com) \\ ${ }^{b}$ Chemistry Division, Institute of Science and Technology, JNT University, Hyderabad, India \\ ${ }^{c}$ Department of Chemistry, Sreenidhi Institute of Science and Technology (Autonomous), \\ Ghatkesar, Hyderabad-501301, Andhra Pradesh, India
}

\section{SUPPORTING INFORMATION}

CONTENTS

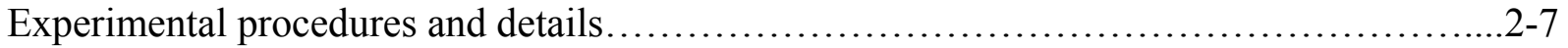

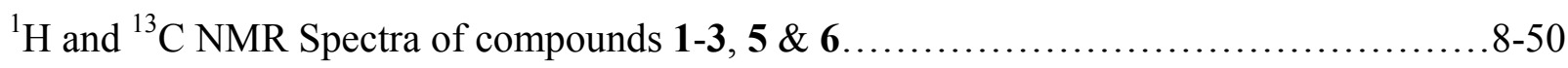


5-(4-bromophenyl)-1,3,4-oxadiazol-2-amine (1): To a stirred solution of ethyl 4bromobenzoate I $(50 \mathrm{~g}, 0.218 \mathrm{~mol})$ in Ethanol $(750 \mathrm{~mL})$ was added hydrazine hydrate $(50 \mathrm{~mL}, 1$ vol) and stirred at reflux temp for $16 \mathrm{~h}$. The solvent was evaporated, the residue was triturated with pet ether to give hydrazide. The hydrazide was taken in in Ethanol (500 mL) and added cyanogen bromide (34 g, $0.327 \mathrm{mmol}$ ) and stirred at RT for $16 \mathrm{~h}$. After completion, the solvent was evaporated and the residue was poured into ice water and filtered the solid to obtain oxadiazole 1 (37.7 g, 72\%, over two steps) as a white solid; m. p. $270-272{ }^{\circ} \mathrm{C}$; IR (KBr) v: 3292 , 3110, $1660 \mathrm{~cm}^{-1} ;{ }^{1} \mathrm{H}$ NMR (DMSO- $\left.d_{6}, 300 \mathrm{MHz}\right): \delta 7.75$ (t, 4H, Phenyl), $7.35\left(\mathrm{~s}, 2 \mathrm{H},-\mathrm{NH}_{2}\right){ }^{13} \mathrm{C}$ NMR (DMSO- $\left.d_{6}, 75 \mathrm{MHz}\right): \delta 163.7,156.6,132.3,126.9,123.7,123.4 ;{ }^{i t-35}{ }^{1} \mathrm{H} \mathrm{NMR}\left(\mathrm{CDCl}_{3}\right): \delta$ 7.25-7.69 (dd, 4H, J = 2.6, 5.6 Hz, Ar-H), 7.75 (s, 2H, NH); ${ }^{13} \mathrm{C}$ NMR $\left(\mathrm{CDCl}_{3}\right)$ : 144-130 (Ar-C). LC-MS: m/z 240.09 (M+H). HRMS. Calcd. for $\mathrm{C}_{8} \mathrm{H}_{7} \mathrm{BrN}_{3} \mathrm{O}: 240.0875$, Found: 240.0879.

2-(4-bromophenyl)-7-hydroxy-5H-[1,3,4]oxadiazolo[3,2-a] pyrimidin-5-one (2): To a stirred solution of 5-(4-bromophenyl)-1,3,4-oxadiazol-2-amine (1) (20 g, $83.2 \mathrm{mmol})$ in Chlorobenzene $(400 \mathrm{~mL})$ was added $\operatorname{Bis}\left(1,3,5\right.$,trichlorophenyl)malonate $(38.4 \mathrm{~g}, 83.2 \mathrm{mmol})$ at $100{ }^{\circ} \mathrm{C}$ and the mixture was refluxed under stirring for $1 \mathrm{~h}$. The reaction mixture was cooled and diluted with pet. ether, solid that precipitated was filtered, washed with pet ether to give pyrimidinone derivative 2 (23.6, 92\%) as a pale yellow solid; m. p. 290-292 ${ }^{\circ} \mathrm{C}$; IR (KBr) v: 3447, 3087, 2923 $\mathrm{cm}^{-1}$; ${ }^{1} \mathrm{H}$ NMR (DMSO- $\left.d_{6}, 300 \mathrm{MHz}\right): \delta 12.03$ (s, 1H, -OH), 7.98-7.87 (m, 4H, Phenyl), 5.36 (s, $1 \mathrm{H}$, pyrimidine); ${ }^{13} \mathrm{C}$ NMR (DMSO- $\left.d_{6}, 75 \mathrm{MHz}\right): \delta 169.3,156.9,156.1,155.2,132.7,128.7$, 127.1, 121.4, 84.7; LC-MS: m/z $308.4(\mathrm{M}+\mathrm{H})$. HRMS. Calcd. for $\mathrm{C}_{11} \mathrm{H}_{7} \mathrm{BrN}_{3} \mathrm{O}_{3}: 308.4012$, Found: 308.4017 .

2-(4-bromophenyl)-7-chloro-5H-[1,3,4] oxadiazolo[3,2-a] pyrimidin-5-one (3): A solution of 2-(4-bromophenyl)-7-hydroxy-5H-[1,3,4] oxadiazolo[3,2-a]pyrimidin-5-one 2 (20 g, $64.8 \mathrm{mmol}$ ) in $\mathrm{POCl}_{3}(100 \mathrm{~mL})$ refluxed under stirring for $2 \mathrm{~h}$. The reaction mixture was cooled and poured into ice water $(500 \mathrm{~mL})$, solid that precipitated was filtered, washed with pet ether to give chloro pyrimidinone derivative 3 (19.9 g, 94\%) as pale yellow solid; m.p 210-212 ${ }^{\circ} \mathrm{C}$; IR (KBr) v: 3422 , 3089, $2921 \mathrm{~cm}^{-1} ;{ }^{1} \mathrm{H}$ NMR (DMSO- $d_{6}, 300 \mathrm{MHz}$ ): $\delta 8.01$ (d, 2H, Phenyl), 7.75 (d, 2H, Phenyl), 6.51 (s, 1H, pyrimidine); ${ }^{13} \mathrm{C}$ NMR (DMSO- $d_{6}, 75 \mathrm{MHz}$ ): $\delta$ 159.1, 157.7, 154.1, 133.1,129.7, 128.9, 119.7, 107.9; LC-MS: m/z $326(\mathrm{M}+\mathrm{H})$. HRMS. Calcd. for $\mathrm{C}_{11} \mathrm{H}_{6} \mathrm{BrClN}_{3} \mathrm{O}_{2}: 326.0016$, Found: 326.0012 . 
4-bromo-N-(4-chloro-2-morpholino-6-oxopyrimidin-1(6H)-yl)benzamide (5a): Off white solid (494 mg, 78\%), m.p. 240-242 ${ }^{\circ} \mathrm{C}$; IR (KBr) v: 3295, 1662, $1541 \mathrm{~cm}^{-1}$; ${ }^{1} \mathrm{H}$ NMR $\left(\mathrm{CDCl}_{3}, 300\right.$ MHz): $\delta 10.24$ (s, 1H, -CO-NH-), 7.62 (d, 2H, $J=8.0$ Hz, Phenyl), 7.47 (d, 2H, $J=8.0 \mathrm{~Hz}$, Phenyl), 6.12 (s, 1H, pyrimidine- $\left.\mathrm{C}_{5} \mathrm{H}\right), 3.78-3.63$ (m, 6H, Morpholine), 3.49 (t, $2 \mathrm{H}, J=13.1 \mathrm{~Hz}$, Morpholine); ${ }^{13} \mathrm{C}$ NMR (DMSO- $\left.d_{6}, 75 \mathrm{MHz}\right): \delta 164.1,160.6,156.8,156.3,131.9,130.0,129.7$, 126.8, 102.5, 65.6, 48.6; HRMS. Calcd. for $\mathrm{C}_{15} \mathrm{H}_{15} \mathrm{BrClN}_{4} \mathrm{O}_{3}$ : 413.0016, Found: 413.0168.

4-bromo-N-(4-chloro-6-oxo-2-(2,2,2 trifluoroethylamino) pyrimidin-1(6H)-yl) benzamide (5b): Off white solid (497 mg, 76\%), m.p. 242-244 ${ }^{\circ} \mathrm{C}$; IR (KBr) v: 3446, 1687, $1667 \mathrm{~cm}^{-1} ;{ }^{1} \mathrm{H}$ NMR (CDCl $3,400 \mathrm{MHz}): \delta 9.02$ (s, 1H, -CO-NH-), $7.82-7.72$ (d, 2H, $J=8.9 \mathrm{~Hz}$, Phenyl), $7.58\left(\mathrm{~d}, 2 \mathrm{H}, J=8.9 \mathrm{~Hz}\right.$, Phenyl), 6.06 (s, 1H, pyrimidine- $\left.\mathrm{C}_{5} \mathrm{H}\right), 5.94\left(\mathrm{t}, 1 \mathrm{H}, J=7.3 \mathrm{~Hz}, \mathrm{CF}_{3} \mathrm{CH}_{2^{-}}\right.$ $\mathrm{NH}), 4.15$ (p, $\left.2 \mathrm{H}, J=8.4 \mathrm{~Hz}, \mathrm{CF}_{3}-\mathrm{CH}_{2}\right) ;{ }^{13} \mathrm{C} \mathrm{NMR}$ (DMSO- $d_{6}, 75 \mathrm{MHz}$ ): $\delta 165.3,158.3,157.5$, 154.2, 131.6, 130.3, 126.6, 125.8, 123.1, 100.7, 41.9; LC-MS : m/z 425.01. HRMS. Calcd. for $\mathrm{C}_{13} \mathrm{H}_{10} \mathrm{BrClF}_{3} \mathrm{~N}_{4} \mathrm{O}_{2}$ : 425.0099, Found: 425.0103 .

N-(2-(allylamino)-4-chloro-6-oxopyrimidin-1(6H)-yl)-4-bromo benzamide (5c): Off white solid (418 mg, 71\%), m.p. 199-201 ${ }^{\circ} \mathrm{C}$; IR (KBr) v: 3293, 1677, $1574 \mathrm{~cm}^{-1} ;{ }^{1} \mathrm{H}$ NMR $\left(\mathrm{CDCl}_{3}\right.$, $400 \mathrm{MHz}$ ): $\delta 9.46$ (s, 1H, -CO-NH-), 7.80-7.68 (d, 2H, Phenyl), 7.56-7.46 (m, 2H, Phenyl), 5.95 (s, $1 \mathrm{H}$, pyrimidine- $\left.\mathrm{C}_{5} \mathrm{H}\right), 5.86\left(\mathrm{~m}, 1 \mathrm{H}, \mathrm{CH}_{2}=\mathrm{C}-\mathrm{H}\right), 5.75(\mathrm{t}, 1 \mathrm{H}, J=5.6 \mathrm{~Hz}$, Allyl-NH), 5.27-5.16 $\left(\mathrm{m}, 2 \mathrm{H}, \mathrm{CH}=\mathrm{CH}_{2}\right), 4.08\left(\mathrm{~m}, 2 \mathrm{H}, \mathrm{CH}_{2}-\mathrm{CH}\right) ;{ }^{13} \mathrm{C} \mathrm{NMR}\left(\mathrm{DMSO}-d_{6}, 75 \mathrm{MHz}\right): \delta 165.3,158.7$, 158.0, 153.6, 134.2, 131.5, 130.5, 130.2, 126.4, 115.3, 98.8, 42.7; LC-MS : m/z 383.03. HRMS. Calcd. for $\mathrm{C}_{14} \mathrm{H}_{13} \mathrm{BrClN}_{4} \mathrm{O}_{2}$ : 383.0014 , Found: 383.0021 .

4-bromo-N-(4-chloro-2-(isopropylamino)-6-oxo pyrimidin-1(6H)-yl) benzamide (5d): Off white solid (428 mg, 72\%), m.p. $143-145{ }^{\circ} \mathrm{C}$; IR (KBr) v: 3335, 1683, 1669, $1567 \mathrm{~cm}^{-1}$; ${ }^{1} \mathrm{H}$ NMR $\left(\mathrm{CDCl}_{3}, 400 \mathrm{MHz}\right): \delta 9.72$ (s, 1H, -CO-NH-), 7.77-7.65 (d, 2H, Phenyl), 7.52-7.41 (d, 2H, Phenyl), 5.89 (s, 1H, pyrimidine- $\left.\mathrm{C}_{5} \mathrm{H}\right), 5.45$ (d, 1H, $J=7.9 \mathrm{~Hz}$, isopropyl-NH), 4.32-4.12 (m, $\left.1 \mathrm{H},\left(\mathrm{CH}_{3}\right)_{2}-\mathrm{C}-\mathrm{H}\right), 1.22\left(\mathrm{~d}, 6 \mathrm{H}, J=6.5 \mathrm{~Hz}, \mathrm{CH}-\left(\mathrm{CH}_{3}\right)_{2}\right) ;{ }^{13} \mathrm{C}$ NMR (DMSO-d $\left.6,75 \mathrm{MHz}\right): \delta 165.8$, $160.9,160.5,152.0,131.8,129.4,128.5,128.0$, 99.8, 44.2, 29.6, 22.3; LC-MS : m/z 385.08. HRMS. Calcd. for $\mathrm{C}_{14} \mathrm{H}_{15} \mathrm{BrClN}_{4} \mathrm{O}_{2}$ : 385.0061 , Found: 385.0066 .

4-bromo-N-(4-chloro-2-(cyclopropylamino)-6-oxo pyrimidin -1(6H)-yl)benzamide (5e): Off white solid (581 mg, 82\%), m.p. 246-248 ${ }^{\circ} \mathrm{C}$; IR (KBr) v: 3343, 1693, 1666, $1567 \mathrm{~cm}^{-1}$; ${ }^{1} \mathrm{H}$ NMR 
$\left(\mathrm{CDCl}_{3}, 400 \mathrm{MHz}\right): \delta 9.62$ (s, 1H, -CO-NH-), 7.69 (d, 2H, J=8.2 Hz, Phenyl), 7.53-7.39 (d, 2H, $J=8.2 \mathrm{~Hz}$, Phenyl), 5.96 (s, 1H, pyrimidine- $\mathrm{C}_{5} \mathrm{H}$ ), 5.82 (s, 1H, cyclopropyl-NH), 2.82 (tq, $1 \mathrm{H}, J$ = 7.0, 3.6 Hz cyclopropyl-CH), 0.95-0.77 (m, 2H, cyclopropyl), 0.64-0.52 (m, 2H, cyclopropyl); ${ }^{13} \mathrm{C}$ NMR (DMSO- $\left.d_{6}, 75 \mathrm{MHz}\right): \delta 165.3,158.3,158.0,155.0,131.4,130.6,130.3,126.4,99.2$, 24.3, 6.2, 5.8; LC-MS : m/z 382.8. HRMS. Calcd. for $\mathrm{C}_{14} \mathrm{H}_{13} \mathrm{BrClN}_{4} \mathrm{O}_{2}$ : 382.9924, Found: 383.9921 .

4-bromo-N-(4-chloro-2-(cyclopentylamino)-6-oxo pyrimidin-1 (6H)-yl)benzamide (5f): Off white solid (470 mg, 74\%), m.p. 186-188 ${ }^{\circ} \mathrm{C}$; IR (KBr) v: 3329, 1684, 1667, $1569 \mathrm{~cm}^{-1}$; ${ }^{1} \mathrm{H}$ NMR $\left(\mathrm{CDCl}_{3}, 400 \mathrm{MHz}\right): \delta 9.74$ (s, 1H, -CO-NH-), 7.71 (d, 2H, $J=8.2 \mathrm{~Hz}$, Phenyl), 7.47 (d, 2H, $J=$ $8.6 \mathrm{~Hz}$, Phenyl), 5.89 (s, 1H, pyrimidine- $\mathrm{C}_{5} \mathrm{H}$ ), 5.58 (d, 1H, $J=7.1 \mathrm{~Hz}$, cyclopentyl-NH), 4.30 (m, 1H, $J=7.1 \mathrm{~Hz}$, cyclopentyl), 2.08 (m, 2H, cyclopentyl), 1.64 (m, 4H, cyclopentyl), 1.48-1.31 (m, 2H, cyclopentyl); ${ }^{13} \mathrm{C}$ NMR (DMSO- $\left.d_{6}, 75 \mathrm{MHz}\right): \delta 165.8,160.9,160.4,152.4,131.8,129.3$, 128.6, 128.0, 99.8, 53.6, 32.7, 23.5; HRMS. Calcd. for $\mathrm{C}_{16} \mathrm{H}_{17} \mathrm{BrClN}_{4} \mathrm{O}_{2}$ : 411.0223. Found: 411.0233.

4-bromo-N-(4-chloro-2-(2-cyclohexylethylamino)-6-oxopyrimidin-1(6H)-yl)benzamide (5g): Off white solid (566 mg, 81\%), m.p. $198-200{ }^{\circ} \mathrm{C}$; IR (KBr) v: $3312,1681,1667,1577 \mathrm{~cm}^{-1} ;{ }^{1} \mathrm{H}$ NMR ( $\left.\mathrm{CDCl}_{3}, 400 \mathrm{MHz}\right): \delta 9.67$ (s, 1H, -CO-NH-), 7.71 (d, 2H, J= 8.5, Phenyl), 7.47 (d, 2H, $J$ $=6.7 \mathrm{~Hz}$, Phenyl), $5.90(\mathrm{~s}, 1 \mathrm{H}$, pyrimidine-C $5 \mathrm{H}), 5.61$ (t, $1 \mathrm{H}, J=5.5 \mathrm{~Hz},-\mathrm{C}-\mathrm{NH}-), 3.43(\mathrm{~m}, 2 \mathrm{H}$, $\left.J=7.6 \mathrm{~Hz},-\mathrm{C}-\mathrm{CH}_{2}-\mathrm{N}-\right), 1.66$ (m, 5H, cyclohexyl), 1.50-1.40 (m, 2H, cyclohexyl), 1.21 (m, 3H, cyclohexyl), 1.03-0.78 (m, 3H, cyclohexyl); ${ }^{13} \mathrm{C}$ NMR (DMSO- $\left.d_{6}, 75 \mathrm{MHz}\right): \delta 165.2,158.7$, 158.0, 153.6, 131.5, 130.6, 130.2, 126.4, 99.3, 36.1, 34.5, 32.6, 26.0, 25.7; LC-MS: m/z 453.15. HRMS. Calcd. for $\mathrm{C}_{19} \mathrm{H}_{23} \mathrm{BrClN}_{4} \mathrm{O}_{2}$ : 453.0687, Found: 453.0691 .

4-bromo-N-(4-chloro-2-(dimethylamino)-6-oxopyrimidin-1(6H)-yl)benzamide (5h): Off white solid (434 mg, 76\%), m.p. 197-199 ${ }^{\circ} \mathrm{C}$; IR (KBr) v: 3207, 1701, 1673, $1575 \mathrm{~cm}^{-1}$; ${ }^{1} \mathrm{H}$ NMR $\left(\mathrm{CDCl}_{3}, 400 \mathrm{MHz}\right): \delta 10.71$ (s, 1H -CO-NH-), 7.63 (d, 2H, $J=8.1 \mathrm{~Hz}$, Phenyl), 7.41 (d, 2H, $J=$ $8.1 \mathrm{~Hz}$, Phenyl), 6.01 (d, $1 \mathrm{H}, J=1.4 \mathrm{~Hz}$, pyrimidine- $\left.\mathrm{C}_{5} \mathrm{H}\right), 3.12\left(\mathrm{~s}, 6 \mathrm{H},-\mathrm{N}\left(\mathrm{CH}_{3}\right)_{2}\right) ;{ }^{13} \mathrm{C}$ NMR (DMSO- $\left.d_{6}, 75 \mathrm{MHz}\right): \delta 164.3,160.7,156.9,156.4,131.9,129.9,129.8,126.7,100.2,40.8$; HRMS. Calcd. for $\mathrm{C}_{13} \mathrm{H}_{13} \mathrm{BrClN}_{4} \mathrm{O}_{2}$ : 370.9910 , Found: 370.9933. 
4-bromo-N-(4-chloro-6-oxo-2-(piperidin-1-yl)pyrimidin-1(6H)-yl) benzamide (5i): Off white solid (495 mg, 78\%), m.p. 177-179 ${ }^{\circ} \mathrm{C}$; IR (KBr) v: 3421, 1706, 1664, $1559 \mathrm{~cm}^{-1}$; ${ }^{1} \mathrm{H}$ NMR $\left(\mathrm{CDCl}_{3}, 400 \mathrm{MHz}\right): \delta 10.65$ (s, 1H, -CO-NH-), 7.61 (d, 2H, J=8.1 Hz, Phenyl), 7.42 (d, 2H, $J=$ $7.9 \mathrm{~Hz}$, Phenyl), 6.05 (s, 1H, pyrimidine- $\mathrm{C}_{5} \mathrm{H}$ ), 3.69-3.36 (m, 4H, piperidine), 1.67-1.49 (m, $6 \mathrm{H}$, piperidine); ${ }^{13} \mathrm{C}$ NMR (DMSO- $\left.d_{6}, 75 \mathrm{MHz}\right): \delta 164.0,163.3,159.7,156.2,131.7,128.9,128.6$, 127.8, 102.0, 49.9, 25.5, 23.9; HRMS. Calculated for $\mathrm{C}_{16} \mathrm{H}_{17} \mathrm{BrClN}_{4} \mathrm{O}_{2}$ : 411.0223, Found: 411.0236 .

4-bromo-N-(4-chloro-2-(ethylamino)-6-oxopyrimidin-1(6H)-yl) benzamide (5j): Off white solid (451 mg, 79\%), m.p. 253-255 ${ }^{\circ} \mathrm{C}$; IR (KBr) v: 3340, 1693, $1666 \mathrm{~cm}^{-1} ;{ }^{1} \mathrm{H} \mathrm{NMR}\left(\mathrm{CDCl}_{3}\right.$, $400 \mathrm{MHz}) \delta$ ppm: 9.62 (s, 1H -CO-NH-), 7.72 (d, 2H, $J=7.9 \mathrm{~Hz}$, Phenyl), 7.54-7.42 (d, 2H, $J=$ $7.9 \mathrm{~Hz}$, Phenyl), 5.92 (s, 1H, pyrimidine- $\mathrm{C}_{5} \mathrm{H}$ ), 5.67 (t, 1H, $J=5.8 \mathrm{~Hz}$ Ethyl-NH), 3.58-3.36 (m, $2 \mathrm{H}$, Ethyl- $\mathrm{CH}_{2}$ ), 1.22 (t, $3 \mathrm{H}, J=7.3 \mathrm{~Hz}$, Ethyl- $\left.\mathrm{CH}_{3}\right) ;{ }^{13} \mathrm{C}$ NMR (DMSO- $d_{6}, 75 \mathrm{MHz}$ ): $\delta 165.3$, 158.7, 158.6, 153.5, 131.5, 130.5, 130.26, 126.4, 98.4, 35.8, 14.35; LC-MS : m/z 371.06. HRMS. Calcd. for $\mathrm{C}_{13} \mathrm{H}_{12} \mathrm{BrClN}_{4} \mathrm{O}_{2} \mathrm{Na}$ : 392.9724, Found: 392.9727.

(S)-N-(2-(3-aminopyrrolidin-1-yl)-4-chloro-6-oxopyrimidin-1(6H)-yl)-4-

bromobenzamide(5k): Off white solid (443 mg, 70\%), m.p. 296-298 ${ }^{\circ} \mathrm{C}$; IR (KBr) v: 3432, 1669, $1591 \mathrm{~cm}^{-1} ;{ }^{1} \mathrm{H}$ NMR (DMSO- $d_{6}, 400 \mathrm{MHz}$ ): $\delta 11.57$ (d, 1H, $\left.J=3.8 \mathrm{~Hz},-\mathrm{CO}-\mathrm{NH}-\right), 8.17$ (s, 3H, $-\mathrm{NH}_{2}$ ), 7.91 (d, 2H, $J=8.4 \mathrm{~Hz}$, Phenyl), 7.85-7.77 (d, 2H, J=8.4 Hz, Phenyl), 6.00 (d, $1 \mathrm{H}, J=1.5 \mathrm{~Hz}$, pyrimidine- $\mathrm{C}_{5} \mathrm{H}$ ), $3.98-3.52$ (m, 6H, Pyrrolidine), 2.19 ( $\mathrm{m}, 1 \mathrm{H}$, Pyrrolidine), 1.98 (m, 1H, Pyrrolidine); ${ }^{13} \mathrm{C}$ NMR (DMSO- $\left.d_{6}, 75 \mathrm{MHz}\right): \delta 165.2,159.8,156.9,153.1,131.7$, 130.0, 126.7, 99.5, 53.4, 52.7, 48.8, 48.2, 28.6; HRMS. Calcd. for $\mathrm{C}_{15} \mathrm{H}_{16} \mathrm{BrClN}_{5} \mathrm{O}_{2}$ : 411.9659, Found: 411.9643 .

4-bromo-N-(2,4-dimorpholino-6-oxopyrimidin-1(6H)-yl) benzamide (6a): Off white solid (543 mg, 77\%), m.p. 290-292 ${ }^{\circ} \mathrm{C}$; IR (KBr) v: 3467, 1697, 1647, $1588 \mathrm{~cm}^{-1}$; ${ }^{1} \mathrm{H}$ NMR (DMSO$\left.d_{6}, 300 \mathrm{MHz}\right): \delta 11.24$ (s, 1H, -CO-NH-), 7.88-7.71 (m, 4H, phenyl), 5.12 (s, 1H, pyrimidine$\mathrm{C}_{5} \mathrm{H}$ ), 3.68-3.38 (m, 14H, morpholine), 3.25 (m, 2H, morpholine); ${ }^{13} \mathrm{C}$ NMR (DMSO- $d_{6}, 75$ $\mathrm{MHz}): \delta 164.2,161.8,159.8,156.2,131.8,130.7,129.6,126.3,78.9,65.8,65.6,48.6,44.1$; HRMS. Calcd. for $\mathrm{C}_{19} \mathrm{H}_{23} \mathrm{BrN}_{5} \mathrm{O}_{4}$ : 464.0933, Found: 464.0926. 
4-bromo-N-(6-oxo-2,4-bis(2,2,2-trifluoro ethylamino) pyrimidin-1(6H)-yl)benzamide (6b): Off white solid (565 mg, 76\%), m.p. 223-225 ${ }^{\circ} \mathrm{C}$; IR (KBr) v: 3312, 1669, $1574 \mathrm{~cm}^{-1}$; ${ }^{1} \mathrm{H}$ NMR (DMSO- $\left.d_{6}, 400 \mathrm{MHz}\right): \delta 10.89(\mathrm{~s}, 1 \mathrm{H},-\mathrm{CO}-\mathrm{NH}-), 8.11\left(\mathrm{t}, 1 \mathrm{H}, J=6.4 \mathrm{~Hz}, \mathrm{CF}_{3} \mathrm{CH}_{2}-\mathrm{NH}\right), 7.92$ (d, 2H, $J=8.2 \mathrm{~Hz}$, Phenyl), 7.79 (d, 2H, $J=8.2 \mathrm{~Hz}$, Phenyl), 7.42 (s, 1H, $\mathrm{CF}_{3} \mathrm{CH}_{2}-\mathrm{NH}$ ), 4.91 (s, $1 \mathrm{H}$, pyrimidine- $\left.\mathrm{C}_{5} \mathrm{H}\right), 4.12\left(\mathrm{~m}, 4 \mathrm{H}, \mathrm{CF}_{3}-\mathrm{CH}_{2}\right) ;{ }^{13} \mathrm{C}$ NMR (DMSO- $\left.d_{6}, 75 \mathrm{MHz}\right): \delta 165.7,161.6$, 159.3, 153.9, 131.4, 130.9, 130.2, 129.3, 129.0, 126.6, 126.2, 123.8, 123.4, 120.6, 77.3, 42.1, 41.8, 41.4, 41.1; LC-MS : m/z 487.95. HRMS. Calcd. for $\mathrm{C}_{15} \mathrm{H}_{13} \mathrm{BrF}_{6} \mathrm{~N}_{5} \mathrm{O}_{2}$ : 488.0151, Found: 488.0155 .

N-(2,4-bis(allylamino)-6-oxopyrimidin-1(6H)-yl)-4-bromo benzamide (6c): Off white solid (437 mg, 70\%), m.p. 218-220 ${ }^{\circ} \mathrm{C}$; IR (KBr) v: 3417, 3387, 1692, $1639 \mathrm{~cm}^{-1}$; ${ }^{1} \mathrm{H}$ NMR (DMSO$\left.d_{6}, 400 \mathrm{MHz}\right): \delta 10.72$ (s, 1H, -CO-NH-), 8.00-7.87 (d, 2H, $J=8.4 \mathrm{~Hz}$, phenyl), 7.83-7.71 (d, $2 \mathrm{H}, J=8.4 \mathrm{~Hz}$, phenyl), 7.61 (s, 1H, allyl-NH-), 6.80 (s, 1H, allyl-NH-), 5.84 (m, 2H, C=CH-C), 5.24-4.97 (m, 4H,-C= $\left.\mathrm{CH}_{2}\right), 4.61\left(\mathrm{~s}, 1 \mathrm{H}\right.$, pyrimidine- $\left.\mathrm{C}_{5} \mathrm{H}\right), 3.85\left(\mathrm{~m}, 2 \mathrm{H}, \mathrm{C}=\mathrm{C}-\mathrm{CH}_{2}-\right), 3.76(\mathrm{~s}$, $\left.2 \mathrm{H}, \mathrm{C}=\mathrm{C}-\mathrm{CH}_{2^{-}}\right) ;{ }^{13} \mathrm{C} \mathrm{NMR}\left(\mathrm{DMSO}-d_{6}, 75 \mathrm{MHz}\right): \delta 165.7,161.6,159.7,153.2,135.4,131.3$, 130.2, 125.9, 115.2, 114.9, 74.5, 42.6; LC-MS : m/z 404.03. HRMS. Calcd. for $\mathrm{C}_{17} \mathrm{H}_{19} \mathrm{BrN}_{5} \mathrm{O}_{2}$ : 404.0716, Found: 404.0720.

N-(2,4-bis(isopropylamino)-6-oxopyrimidin-1(6H)-yl)-4-bromobenzamide (6d): Off white solid (424 mg, 69\%), m.p. 209-210 ${ }^{\circ} \mathrm{C}$; IR (KBr) v: 3308, 1674, $1581 \mathrm{~cm}^{-1} ;{ }^{1} \mathrm{H}$ NMR (DMSO- $d_{6}$, $300 \mathrm{MHz}$ ): $\delta 10.51$ (s, 1H, -CO-NH-), 7.87 (d, 2H, J=7.8 Hz, phenyl), 7.71 (d, 2H, J= 7.9 Hz, Phenyl), $6.81\left(\mathrm{~s}, 1 \mathrm{H}\right.$, isopropyl-NH), $5.76(\mathrm{~s}, 1 \mathrm{H}$, isopropyl- $\mathrm{NH}), 4.91\left(\mathrm{~s}, 1 \mathrm{H}\right.$, pyrimidine- $\left.\mathrm{C}_{5} \mathrm{H}\right)$, 3.91 (m, 1H, isopropyl), 1.34 (d, $6 \mathrm{H}, J=6.8 \mathrm{~Hz}$, isopropyl), 1.28 (d, $6 \mathrm{H}, J=6.6 \mathrm{~Hz}$, isopropyl); ${ }^{13} \mathrm{C}$ NMR (DMSO- $\left.d_{6}, 75 \mathrm{MHz}\right): \delta 167.1,163.8,158.9,152.1,134.3,132.1,129.7,124.6,79.2$, 43.2, 41.8, 28.1, 27.2, 22.7, 22.4; LC-MS: m/z 408.16. HRMS. Calcd. for $\mathrm{C}_{17} \mathrm{H}_{23} \mathrm{BrN}_{5} \mathrm{O}_{2}$ : 408.0951, Found: 413.0946.

N-(2,4-bis(cyclopropylamino)-6-oxopyrimidin-1(6H)-yl)-4-bromobenzamide (6e): Off white solid (500 mg, 82\%), m.p. 201-203 ${ }^{\circ} \mathrm{C}$; IR (KBr) v: 3302, 1670, $1588 \mathrm{~cm}^{-1}$; ${ }^{1} \mathrm{H}$ NMR (DMSO- $d_{6}$, $400 \mathrm{MHz}$ ): $\delta 10.59$ (s, 1H, -CO-NH-), 7.91 (d, 2H, $J=7.9 \mathrm{~Hz}$, phenyl), 7.75 (d, 2H, $J=8.0 \mathrm{~Hz}$, Phenyl), 7.33 (s, 1H, cyclopropyl-NH), 6.83 (s, 1H, cyclopropyl-NH), 4.88 (s, 1H, pyrimidine$\left.\mathrm{C}_{5} \mathrm{H}\right), 2.68$ (m, 1H, cyclopropyl), 2.35 (m, 1H, cyclopropyl), 0.76-0.58 (m, 4H, cyclopropyl), 
0.57-0.41 (m, 4H, cyclopropyl); ${ }^{13} \mathrm{C}$ NMR (DMSO- $d_{6}, 75 \mathrm{MHz}$ ): $\delta 165.7,162.7,159.6,154.3$, 131.3, 131.2, 130.3, 125.9, 75.4, 24.0, 23.4, 6.5, 6.3, 5.9; LC-MS: m/z 404.03. HRMS. Calcd. for $\mathrm{C}_{17} \mathrm{H}_{19} \mathrm{BrN}_{5} \mathrm{O}_{2}: 404.0624$, Found: 404.0619.

N-(2,4-bis(cyclopentylamino)-6-oxopyrimidin-1(6H)-yl)-4-bromobenzamide (6f): Off white solid (559 mg, 79\%), m.p. 206-208 ${ }^{\circ} \mathrm{C}$; IR (KBr) v: 3355, 1654, $1590 \mathrm{~cm}^{-1}$; ${ }^{1} \mathrm{H}$ NMR (DMSO- $d_{6}$, $400 \mathrm{MHz}$ ): $\delta 10.53$ (s, 1H, -CO-NH-), 7.93 (d, 2H, $J=7.8 \mathrm{~Hz}$, Phenyl), 7.76 (d, 2H, $J=7.9 \mathrm{~Hz}$, Phenyl), 6.98 (s, 1H,cyclopentyl-NH), 6.52 (s, 1H, cyclopentyl-NH), 4.59 (s, 1H, pyrimidine$\left.\mathrm{C}_{5} \mathrm{H}\right), 4.22$ (m, 2H, cyclopentyl), 1.87 (m, 4H, cyclopentyl), $1.74-1.39$ (m, $12 \mathrm{H}$, cyclopentyl); ${ }^{13} \mathrm{C}$ NMR (DMSO- $\left.d_{6}, 75 \mathrm{MHz}\right): \delta 165.8,161.2,159.5,153.0,131.3,131.2,130.2,125.8,74.4,52.3$, 32.4, 31.6, 23.5; Calcd. for $\mathrm{C}_{21} \mathrm{H}_{27} \mathrm{BrN}_{5} \mathrm{O}_{2}$ : 460.1348, Found: 460.1339 .

N-(2,4-bis(2-cyclohexylethylamino)-6-oxopyrimidin-1(6H)-yl)-4-bromobenzamide (6g): Off white solid (518 mg, 62\%), m.p. 186-188 ${ }^{\circ} \mathrm{C}$; IR (KBr) v: $3431,1648,1582 \mathrm{~cm}^{-1}$; ${ }^{1} \mathrm{H}$ NMR (DMSO- $\left.d_{6}, 300 \mathrm{MHz}\right): \delta 11.12$ (s, $\left.1 \mathrm{H},-\mathrm{CO}-\mathrm{NH}-\right), 7.91-7.75$ (m, 4H, phenyl), $5.11(\mathrm{~s}, 1 \mathrm{H}$, pyrimidine- $\mathrm{C}_{5} \mathrm{H}$ ), 2.65-2.59 (m, $4 \mathrm{H},-\mathrm{CH}_{2}$ ) $)$ 1.69-1.59 (m, $10 \mathrm{H}$, cyclohexyl), 1.55-1.42 (m, $16 \mathrm{H}$, cyclohexyl); ${ }^{13} \mathrm{C}$ NMR (DMSO- $d_{6}, 75 \mathrm{MHz}$ ): $\delta 165.2,163,162.2,154.5,131.8,131.5,129.8$, 126.2, 78.3, 39.7, 36.8, 35.6, 35.2, 33.5 33.1, 33.0, 29.1, 28.9, 28.2, 27.9; LC-MS: m/z 544.31. HRMS. Calcd. for $\mathrm{C}_{27} \mathrm{H}_{39} \mathrm{BrN}_{5} \mathrm{O}_{2}$ : 544.3204, Found: 544.3198 .

N-(2,4-bis(dimethylamino)-6-oxopyrimidin-1(6H)-yl)-4-bromobenzamide (6h): Off white solid (436 mg, 75\%), m.p. 250-252 ${ }^{\circ} \mathrm{C}$; IR (KBr) v: 3448, 1686, 1643, $1591 \mathrm{~cm}^{-1} ;{ }^{1} \mathrm{H}$ NMR (DMSO-d $d_{6}, 400$ MHz): $\delta: 11.16$ (s, 1H, -CO-NH-), 7.87 (d, 2H, $J=8.4$ Hz, Phenyl), 7.78 (d, 2H, $J=8.2 \mathrm{~Hz}$, phenyl), 4.84 (s, $1 \mathrm{H}$, pyrimidine- $\mathrm{C}_{5} \mathrm{H}$ ), 2.99 (s, 3H, Dimethyl), 2.92 (s, 9H, Dimethyl); ${ }^{13} \mathrm{C}$ NMR (DMSO- $\left.d_{6}, 75 \mathrm{MHz}\right): \delta 164.3,161.6,160.2,156.2,131.7,130.7,129.7$, 126.2, 76.5, 40.3, 37.0; HRMS. Calcd. for $\mathrm{C}_{15} \mathrm{H}_{19} \mathrm{BrN}_{5} \mathrm{O}_{2}: 380.0722$, Found: 380.0733 .

4-bromo-N-(6-oxo-2,4-di(piperidin-1-yl)pyrimidin-1(6H)-yl)benzamide (6i): Off white solid (516 mg, 73\%), m.p. 259-261 ${ }^{\circ} \mathrm{C}$; IR (KBr) v: 3447, 1703, 1646, $1591 \mathrm{~cm}^{-1}$; ${ }^{1} \mathrm{H}$ NMR (DMSO$\left.d_{6}, 400 \mathrm{MHz}\right): \delta 11.21$ (s, 1H, -CO-NH-), 7.92-7.73 (m, 4H, phenyl), 5.05 (s, 1H, pyrimidine$\left.\mathrm{C}_{5} \mathrm{H}\right), 3.50$ (m, 4H, Piperidine), 3.43-3.33 (m, 2H, Piperidine), 3.25 (m, 2H, Piperidine), 1.721.30 (m, 12H, Piperidine); ${ }^{13} \mathrm{C}$ NMR (DMSO- $\left.d_{6}, 75 \mathrm{MHz}\right): \delta 163.9,162.1,159.4,156.5,131.7$, 
130.9, 129.6, 126.2, 77.9, 49.1, 44.8, 25.1, 24.0, 23.8; HRMS. Calcd. for $\mathrm{C}_{21} \mathrm{H}_{27} \mathrm{BrN}_{5} \mathrm{O}_{2}$ : 460.1348. Found: 460.1334.

N-(2,4-bis(ethylamino)-6-oxopyrimidin-1(6H)-yl)-4-bromo benzamide (6j): Off white solid (421 mg, 73\%), m.p. $220-222{ }^{\circ} \mathrm{C}$; IR (KBr) v: 3317, 1685, $1652 \mathrm{~cm}^{-1}$; ${ }^{1} \mathrm{H}$ NMR (DMSO- $d_{6}, 300$ MHz): $\delta 10.63$ (s, 1H, -CO-NH-), 8.01 (t, $\left.1 \mathrm{H}, J=6.3 \mathrm{~Hz}, \mathrm{CH}_{3} \mathrm{CH}_{2}-\mathrm{NH}\right), 7.85$ (d, 2H, $J=8.0 \mathrm{~Hz}$, Phenyl), 7.61 (d, $2 \mathrm{H}, J=7.9 \mathrm{~Hz}$, Phenyl), 7.36 (s, $1 \mathrm{H}, \mathrm{CH}_{3} \mathrm{CH}_{2}-\mathrm{NH}$ ), 5.04 (s, $1 \mathrm{H}$, pyrimidine$\mathrm{C}_{5} \mathrm{H}$ ), 3.67-3.41 (m, 4H, $\mathrm{CH}_{3}-\mathrm{CH}_{2}$ ), 1.45-1.21 (m, 6H, Ethyl- $\mathrm{CH}_{3}$ ); ${ }^{13} \mathrm{C}$ NMR (DMSO- $d_{6}, 75$ MHz): $\delta 163.1,161.6,158.9,153.4,131.1,130.5,129.2,128.7,128.3,126.4,126.2,123.4$, 123.1, 120.6, 75.3, 35.9, 35.1, 14.38, 14.32; LC-MS : $\mathrm{m} / \mathrm{z}$ 380.14. HRMS. Calcd. for $\mathrm{C}_{15} \mathrm{H}_{19} \mathrm{BrN}_{5} \mathrm{O}_{2}: 380.0730$, Found: 380.0727 .

N-(2,4-bis((S)-3-aminopyrrolidin-1-yl)-6-oxopyrimidin-1 (6H)-yl)-4-bromobenzamide (6k): Off white solid (439 mg, 62\%), m.p. 330-332 ${ }^{\circ} \mathrm{C}$; IR (KBr) v, $\mathrm{cm}^{-1}: 3435,1681,1645,1560 ;{ }^{1} \mathrm{H}$ NMR ( $\left.\mathrm{D}_{2} \mathrm{O}, 400 \mathrm{MHz}\right) \delta$ ppm: 7.89-7.72 (m, 4H, phenyl), 4.17 - 3.48 (m, 10H, Pyrrolidine), 2.52-2.28 (m, 2H, Pyrrolidine), 2.27-2.00 ( $\mathrm{m}, 2 \mathrm{H}$, Pyrrolidine); ${ }^{13} \mathrm{C}$ NMR (DMSO- $d_{6}, 75 \mathrm{MHz}$ ): $\delta 164.2,160.7,159.7,153.1,131.4,129.9,126.8,78.9,53.4,53.2,52.7,52.6,48.8,48.2,28.3$; HRMS. Calcd. for $\mathrm{C}_{19} \mathrm{H}_{25} \mathrm{BrN}_{7} \mathrm{O}_{2}: 462.0777$, Found: 462.0761. 


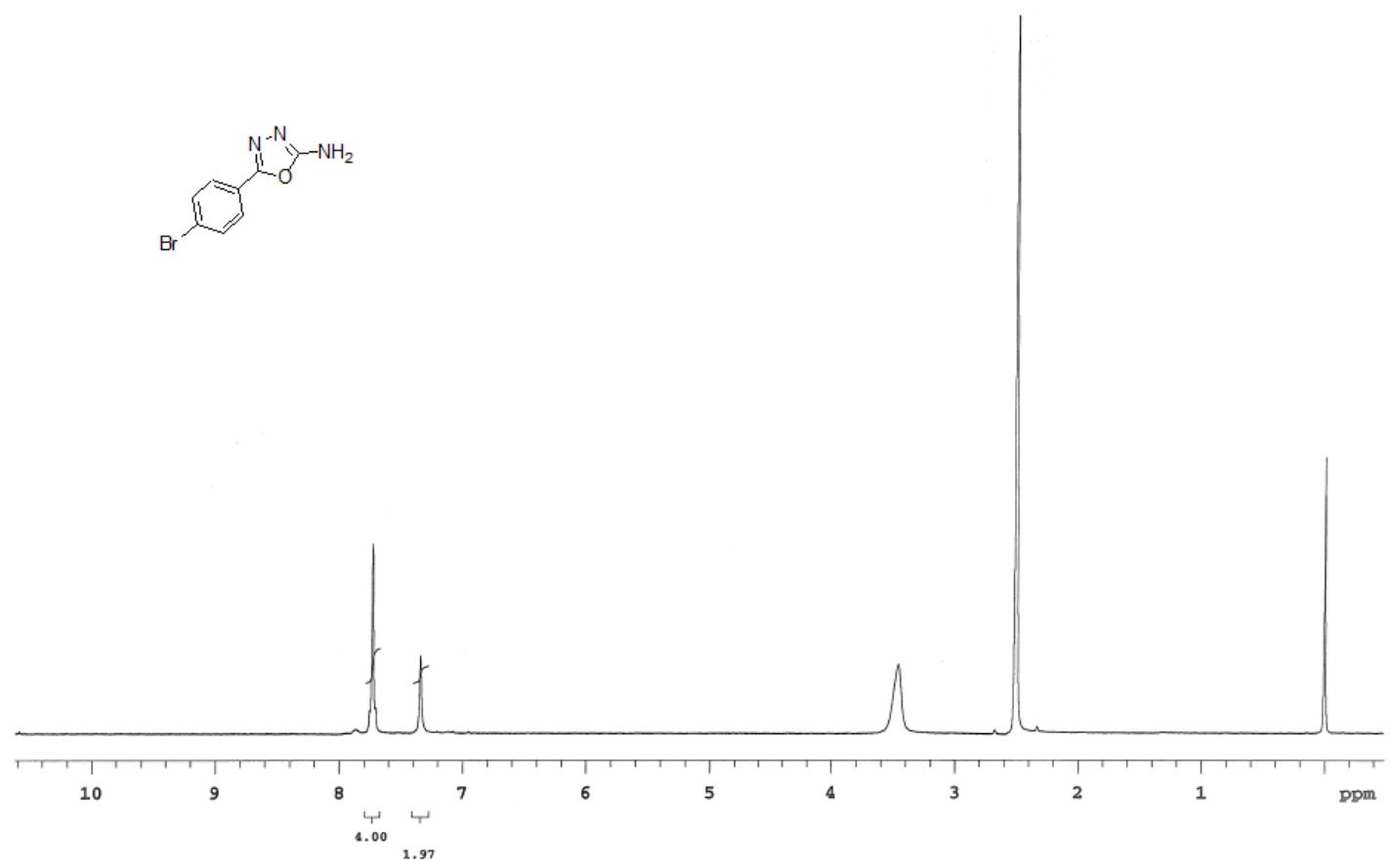

Figure S1. ${ }^{1} \mathrm{H}$ NMR Spectrum of 1 (300 MHz, DMSO- $\left.d_{6}, 298 \mathrm{~K}\right)$. 


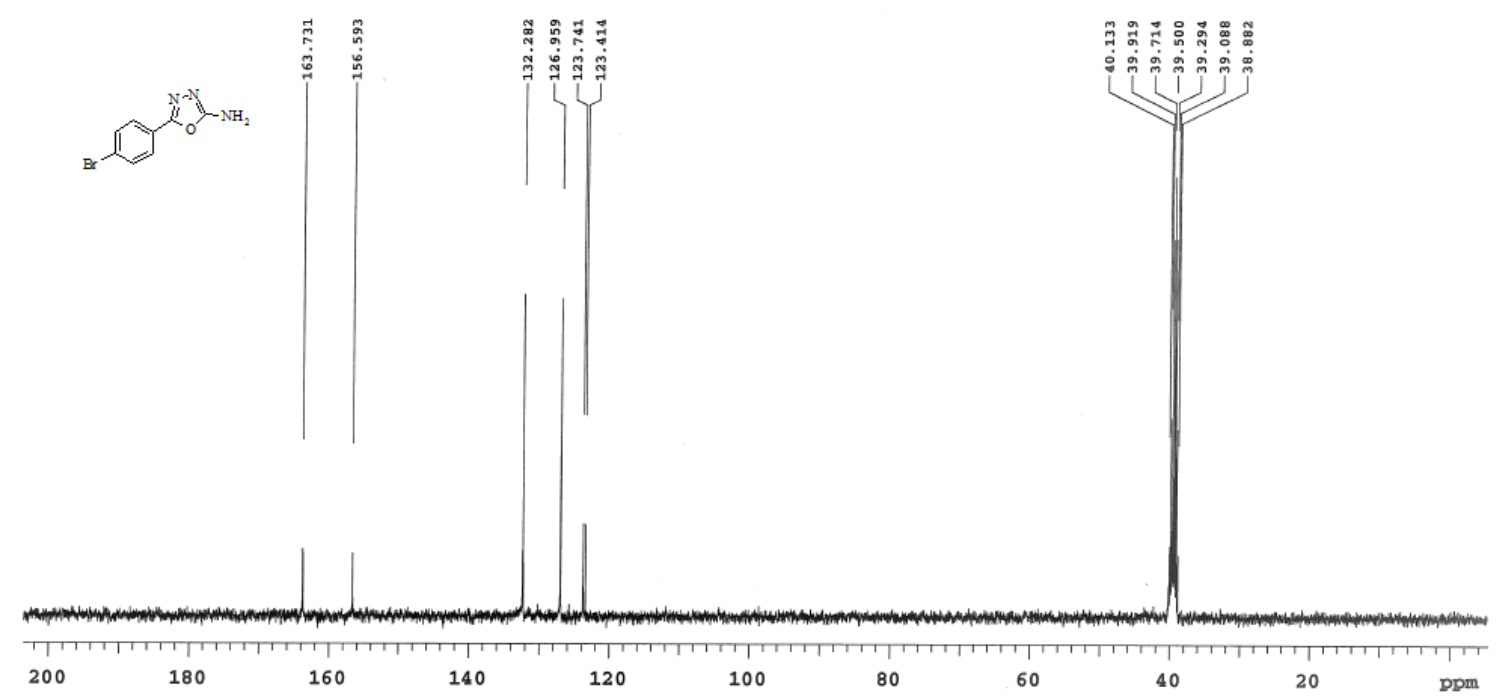

Figure S2. ${ }^{13} \mathrm{C}$ NMR Spectrum of 1 (75 MHz, DMSO- $\left.d_{6}, 298 \mathrm{~K}\right)$. 


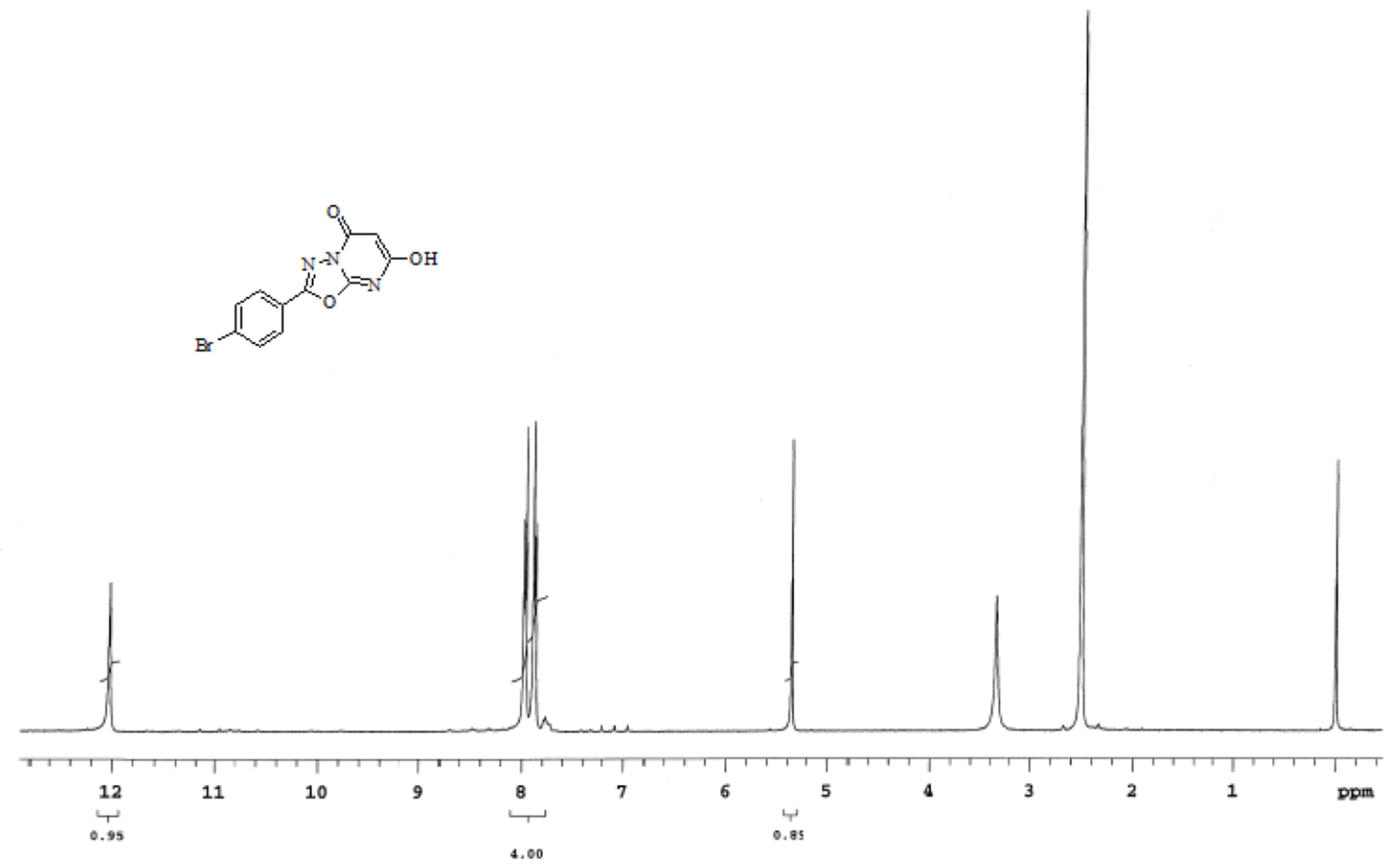

Figure S3. ${ }^{1} \mathrm{H}$ NMR Spectrum of $2\left(300 \mathrm{MHz}, \mathrm{DMSO}-d_{6}, 298 \mathrm{~K}\right)$. 


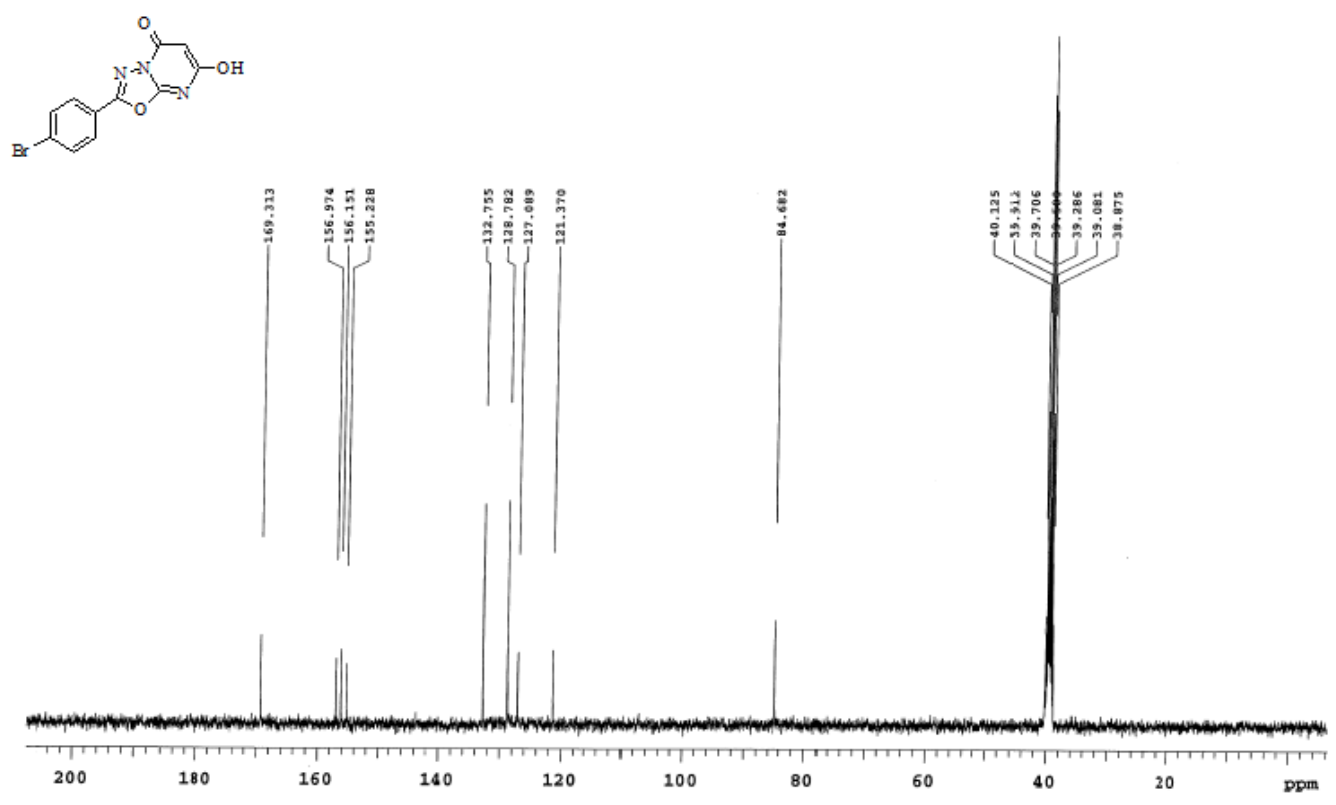

Figure S4. ${ }^{13} \mathrm{C}$ NMR Spectrum of 2 (75 MHz, DMSO- $\left.d_{6}, 298 \mathrm{~K}\right)$. 


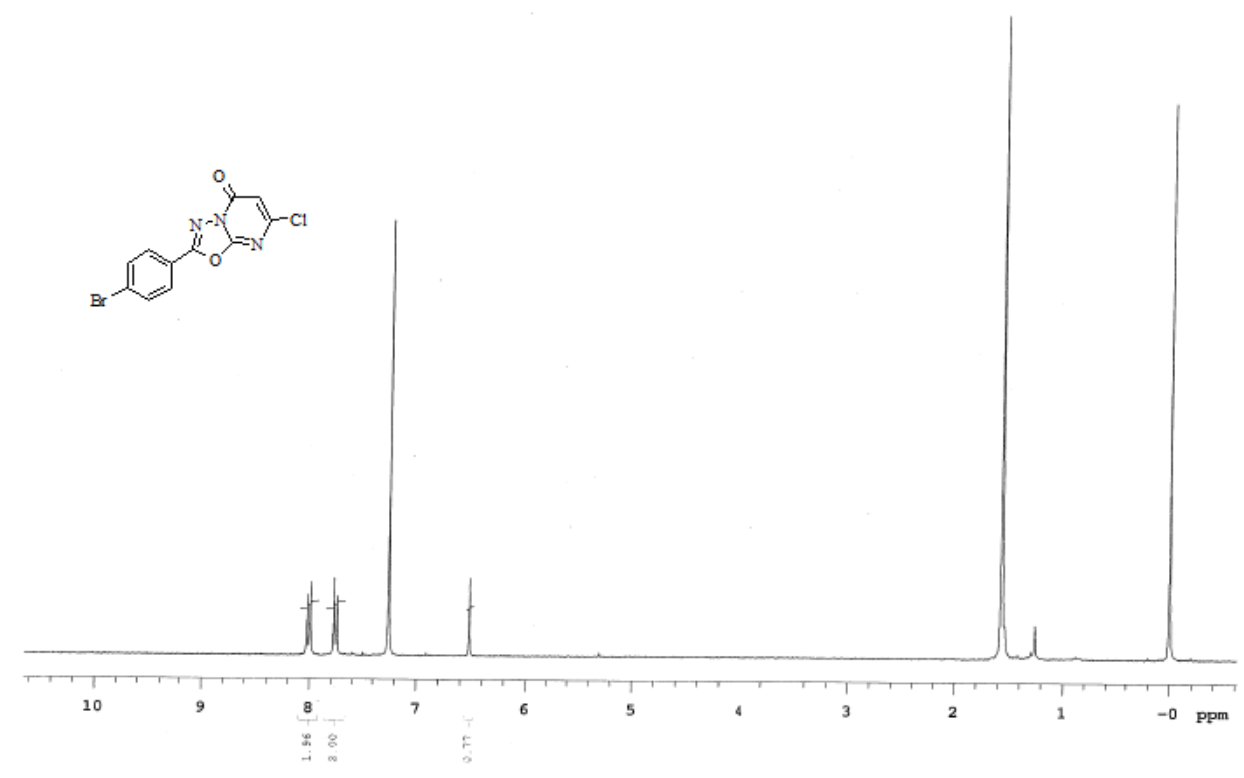

Figure S5. ${ }^{1} \mathrm{H}$ NMR Spectrum of $3\left(300 \mathrm{MHz}, \mathrm{CDCl}_{3}, 298 \mathrm{~K}\right)$. 


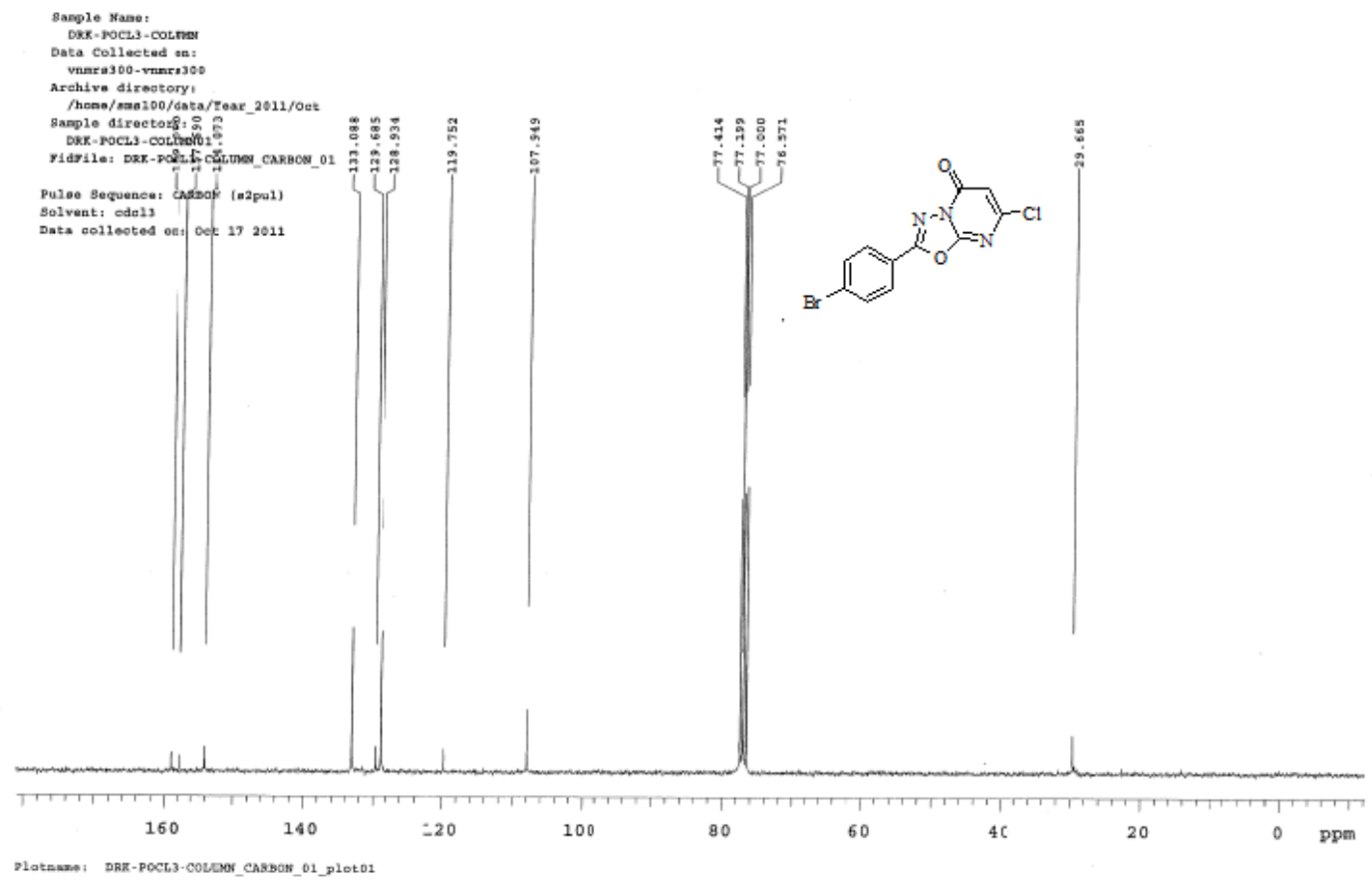

Figure S6. ${ }^{1} \mathrm{H}$ NMR Spectrum of $3\left(75 \mathrm{MHz}, \mathrm{CDCl}_{3}, 298 \mathrm{~K}\right)$. 


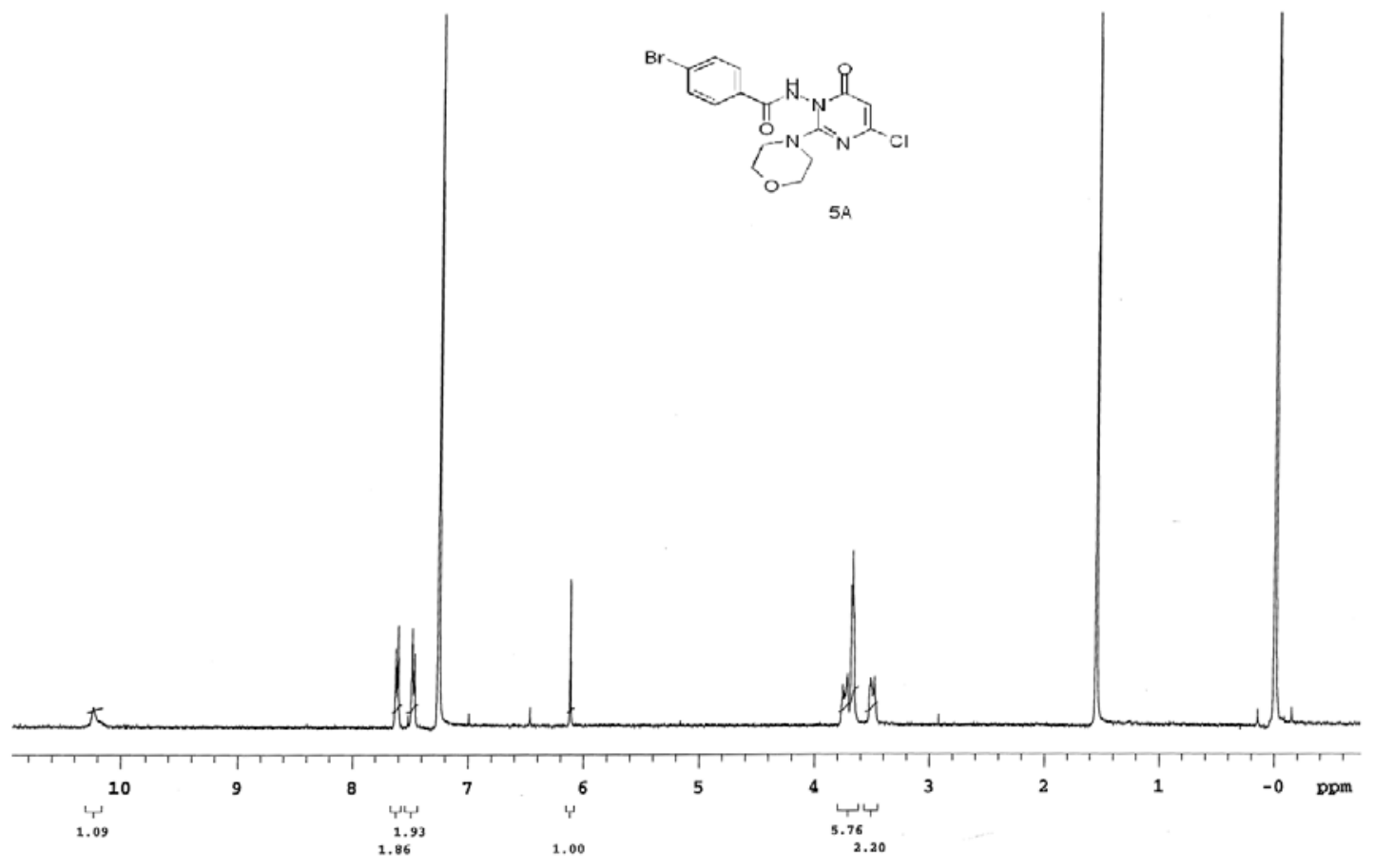

Figure S7. ${ }^{1} \mathrm{H}$ NMR Spectrum of $\mathbf{5 A}\left(300 \mathrm{MHz}, \mathrm{CDCl}_{3}, 298 \mathrm{~K}\right)$. 

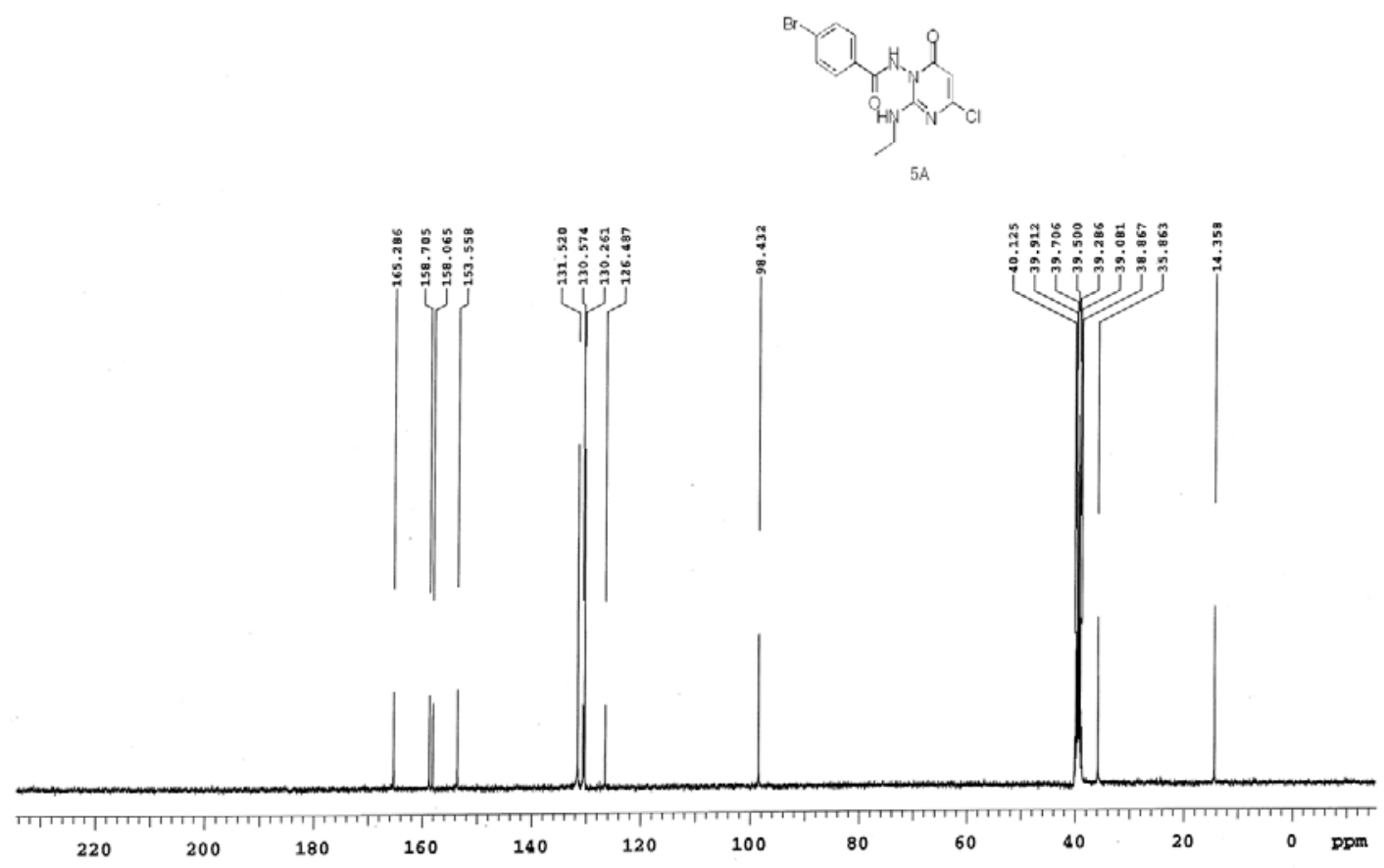

Figure S8. ${ }^{13} \mathrm{C}$ NMR Spectrum of $\mathbf{5 A}\left(75 \mathrm{MHz}, \mathrm{DMSO}-d_{6}, 298 \mathrm{~K}\right)$. 


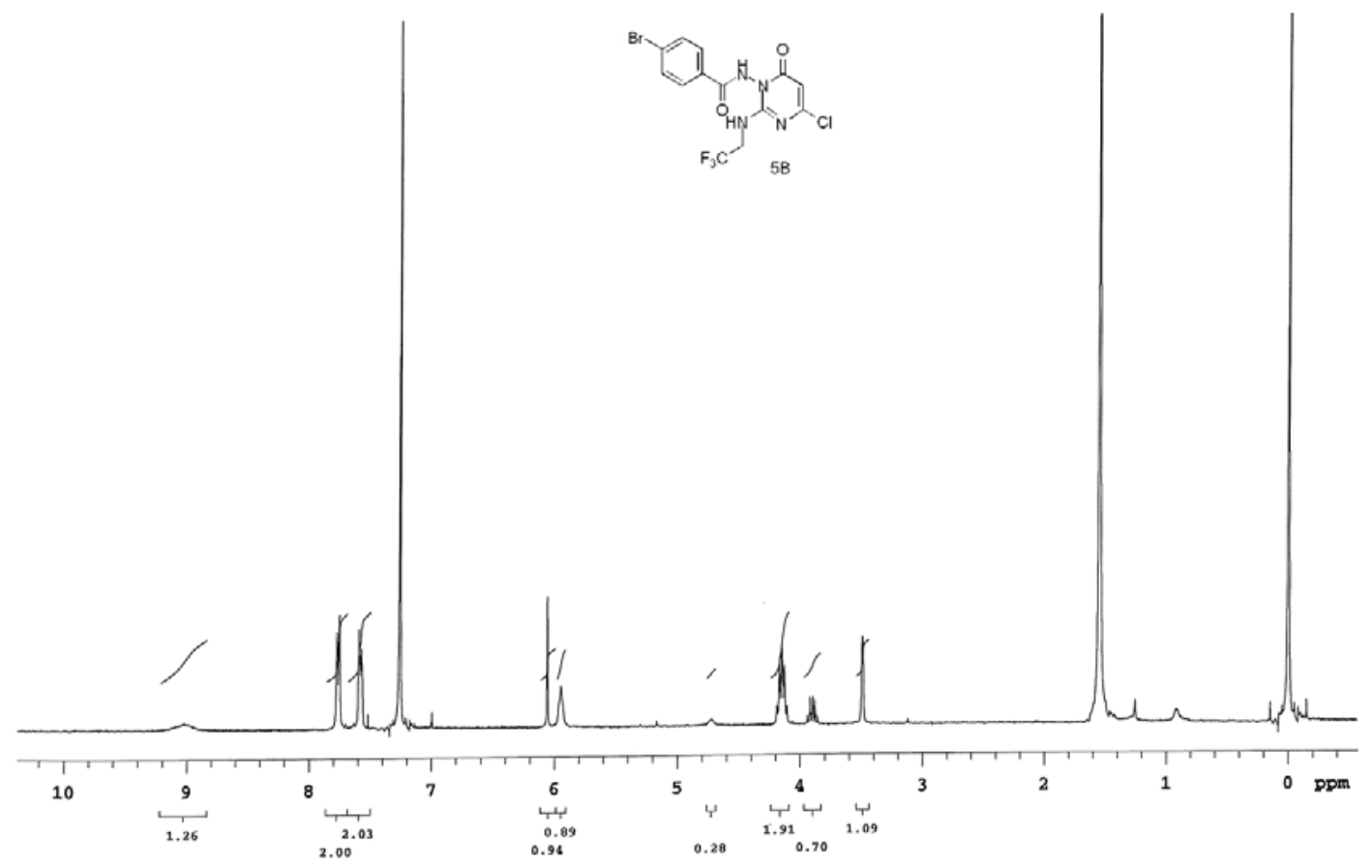

Figure S9. ${ }^{1} \mathrm{H}$ NMR Spectrum of $\mathbf{5 b}\left(400 \mathrm{MHz}, \mathrm{CDCl}_{3}, 298 \mathrm{~K}\right)$. 


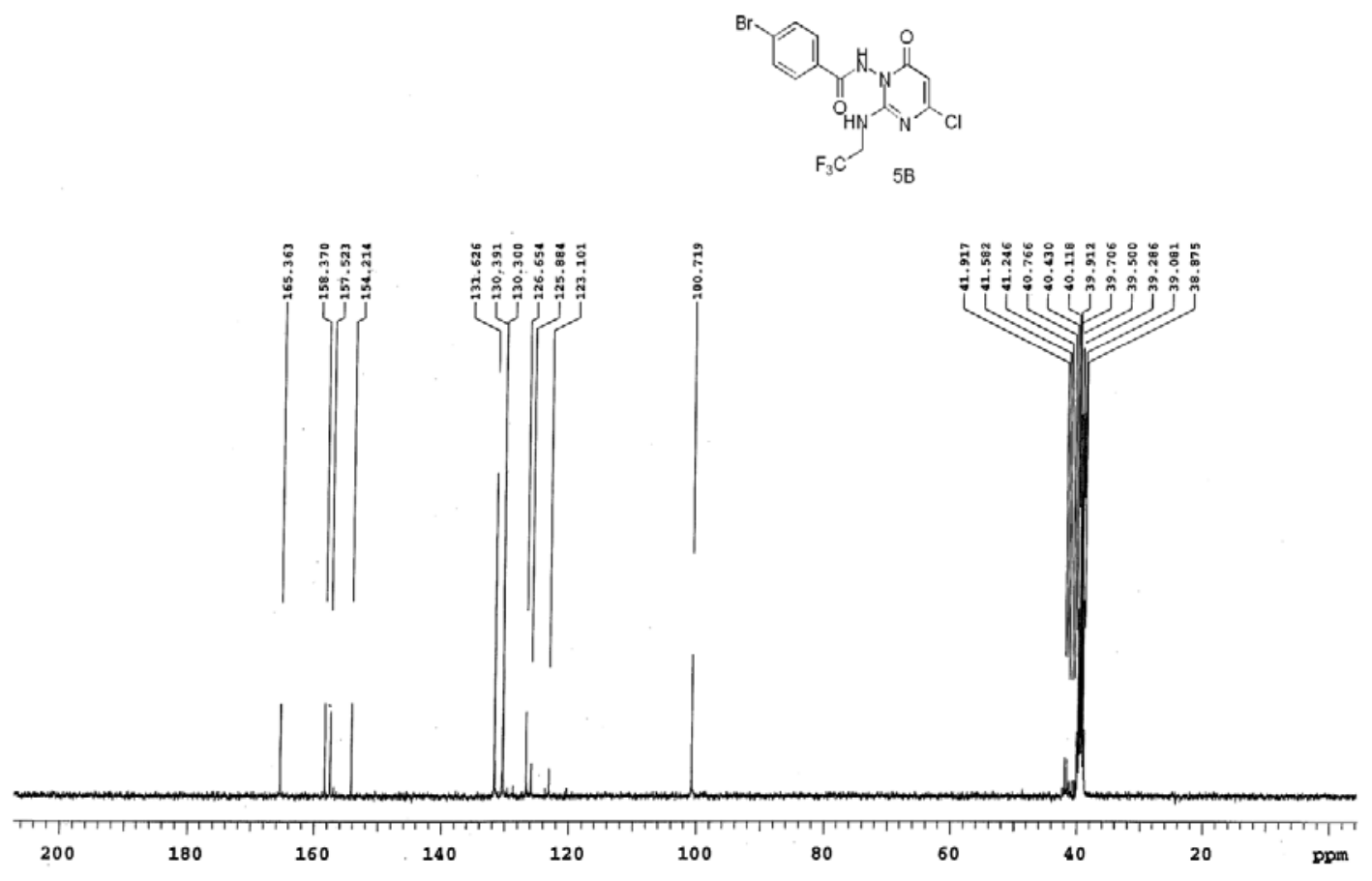

Figure S10. ${ }^{13} \mathrm{C}$ NMR Spectrum of $\mathbf{5 b}\left(75 \mathrm{MHz}, \mathrm{DMSO}-d_{6}, 298 \mathrm{~K}\right)$. 


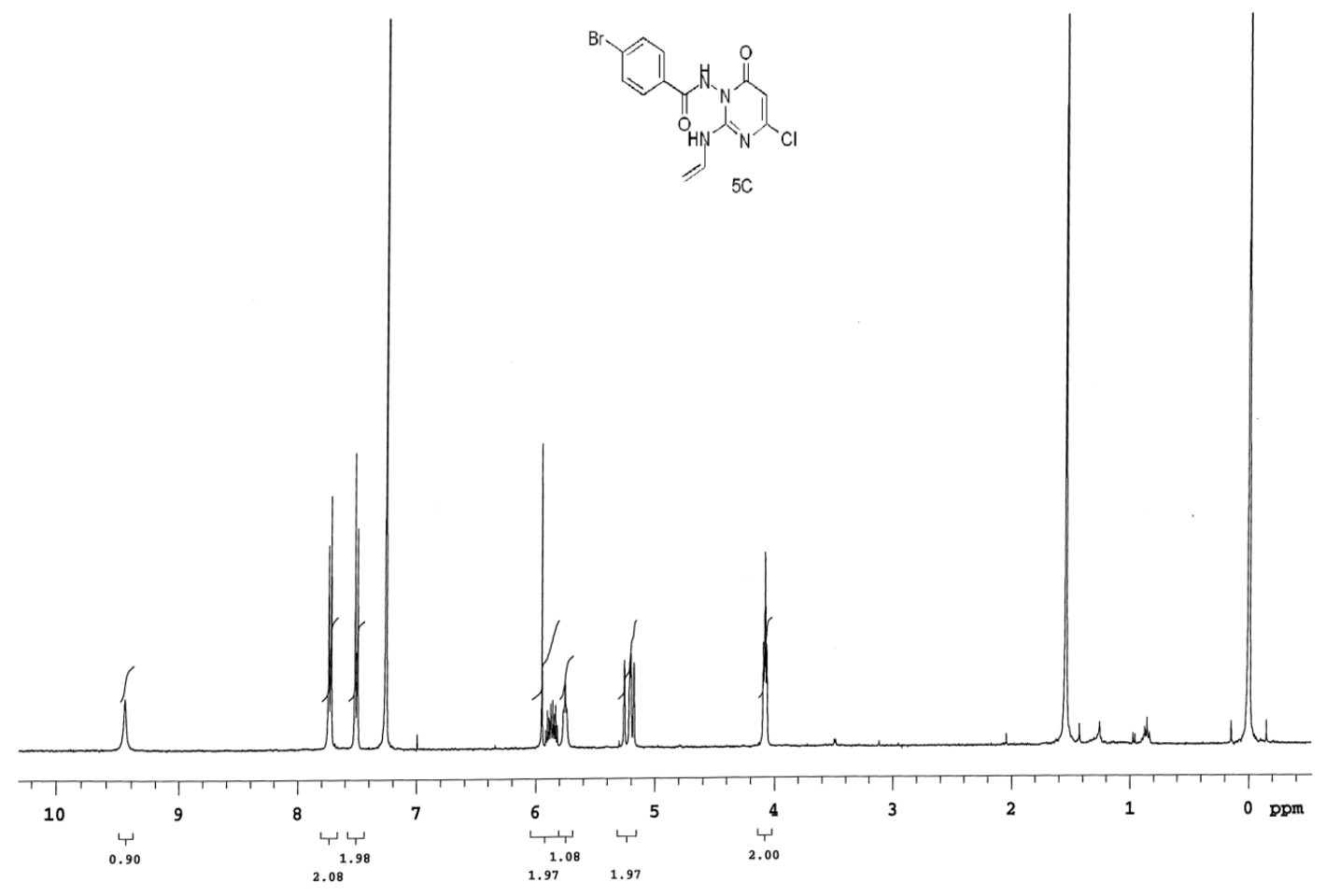

Figure S11. ${ }^{1} \mathrm{H}$ NMR Spectrum of $\mathbf{5 c}\left(400 \mathrm{MHz}, \mathrm{CDCl}_{3}, 298 \mathrm{~K}\right)$. 


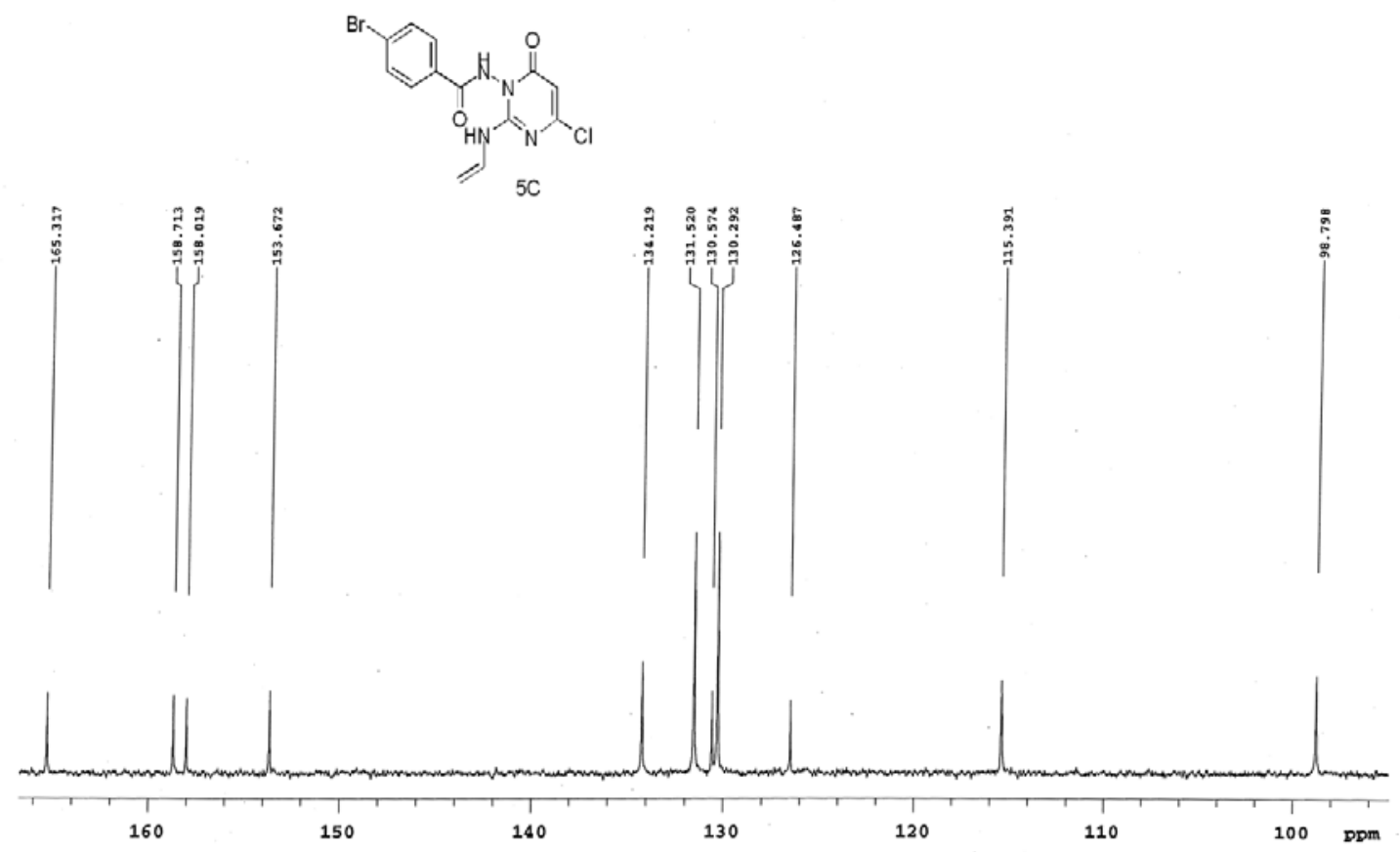

Figure S12. ${ }^{13} \mathrm{C}$ NMR Spectrum of $\mathbf{5 c}(75 \mathrm{MHz}$, DMSO-d6, $298 \mathrm{~K}$ ). 


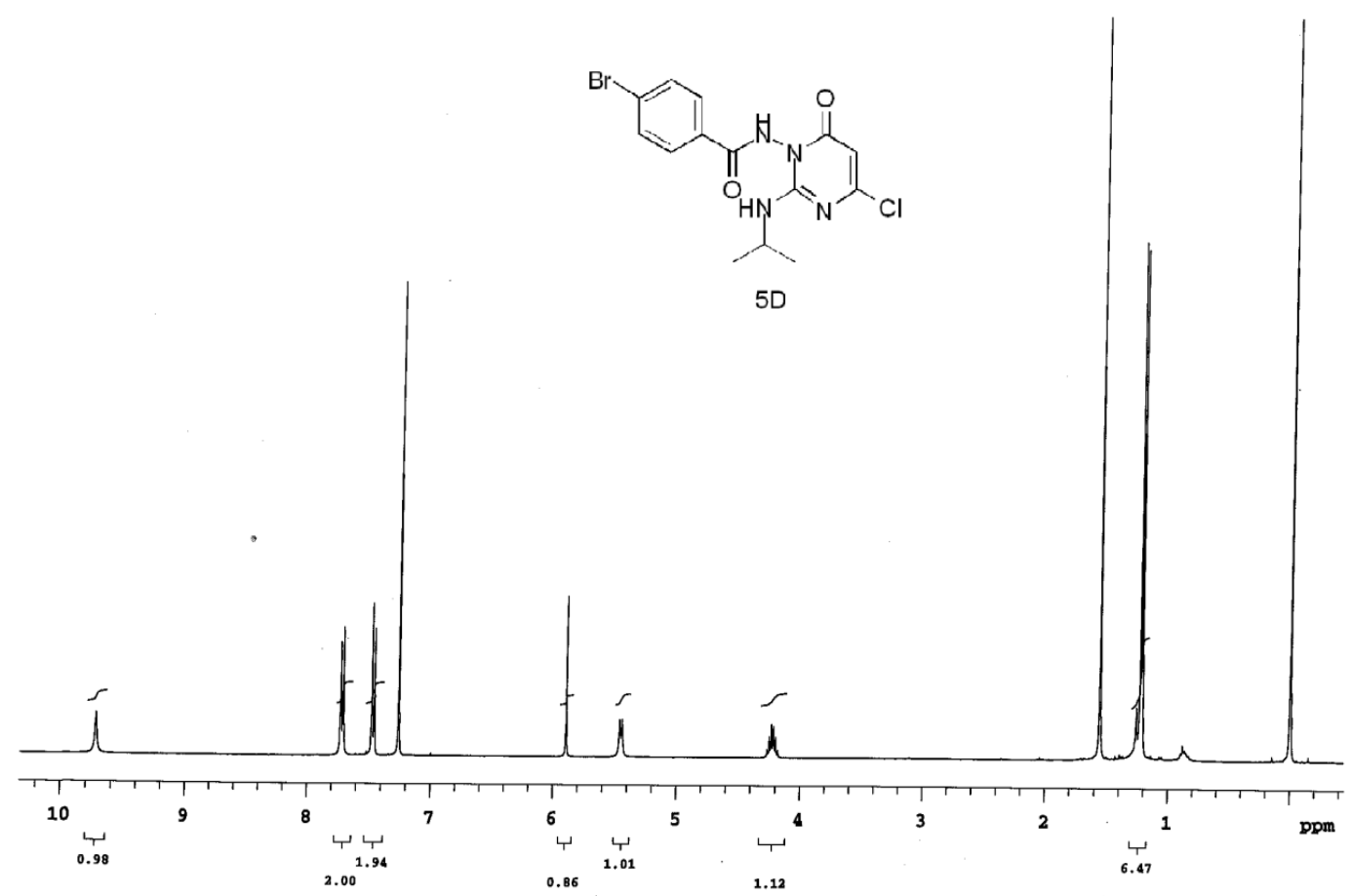

Figure S13. ${ }^{13} \mathrm{H}$ NMR Spectrum of $\mathbf{5 d}\left(400 \mathrm{MHz}, \mathrm{CDCl}_{3}, 298 \mathrm{~K}\right)$. 


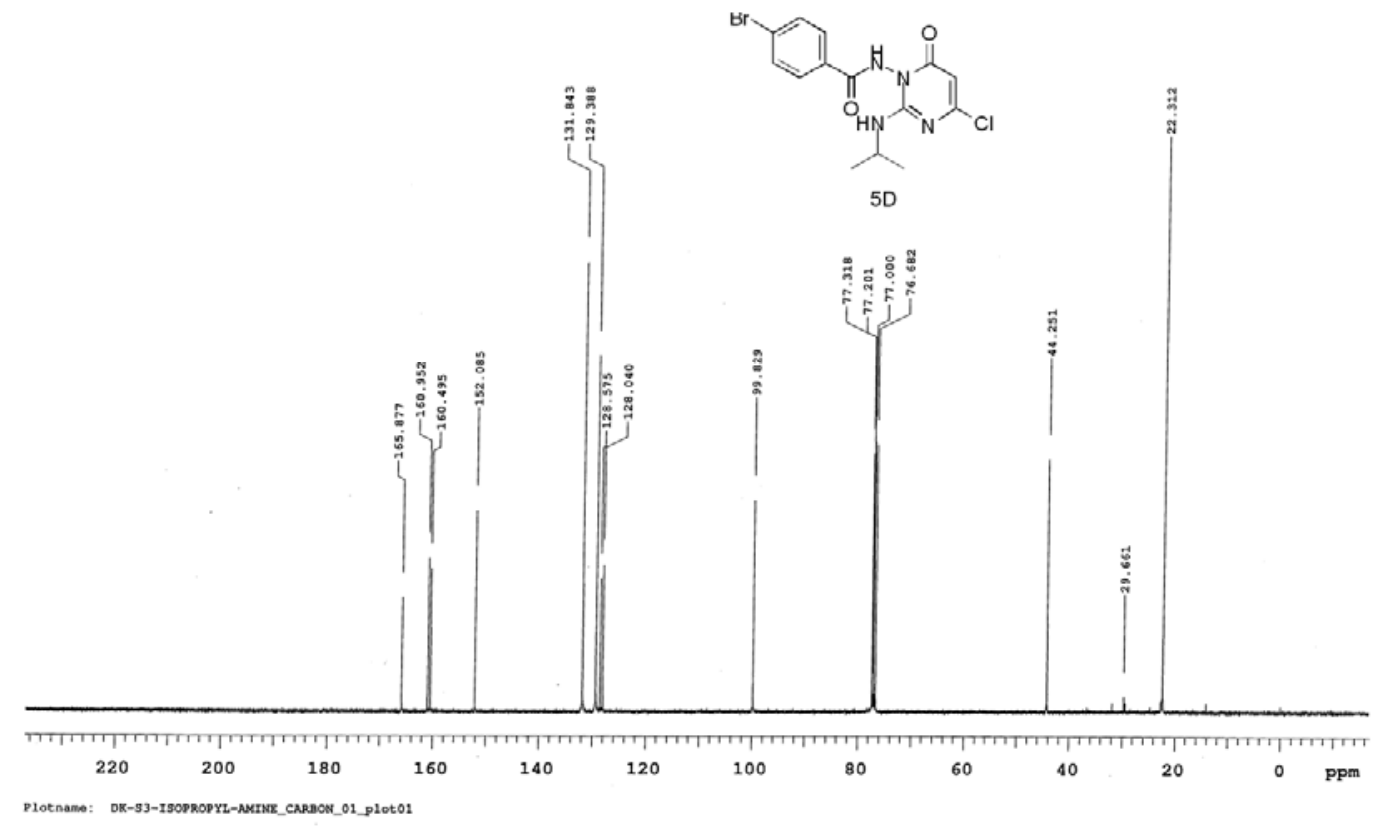

Figure S14. ${ }^{13} \mathrm{C}$ NMR Spectrum of $\mathbf{5 d}\left(75 \mathrm{MHz}, \mathrm{CDCl}_{3}, 298 \mathrm{~K}\right)$. 


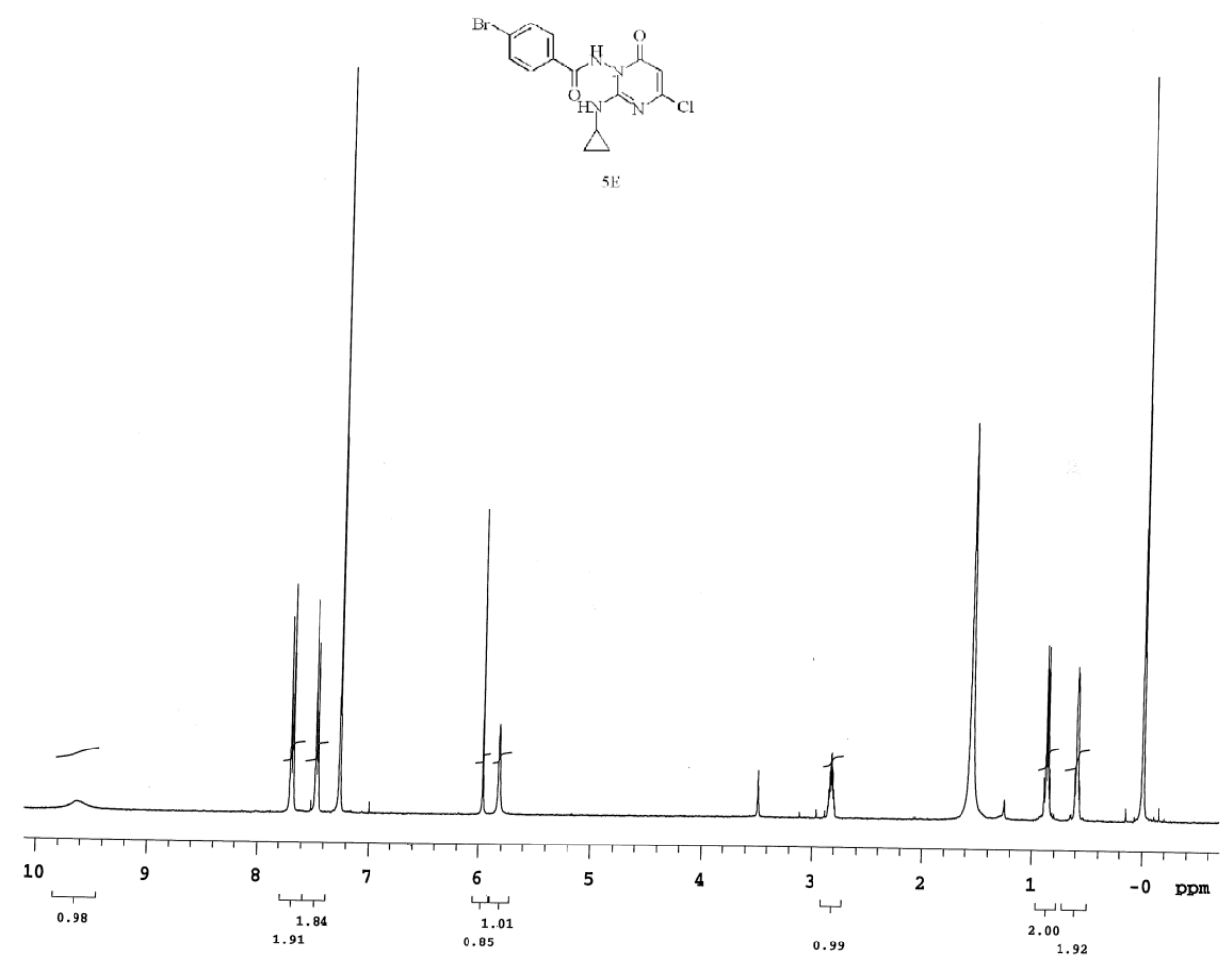

Figure S15. ${ }^{1} \mathrm{H}$ NMR Spectrum of $\mathbf{5 e}\left(400 \mathrm{MHz}, \mathrm{CDCl}_{3}, 298 \mathrm{~K}\right)$. 


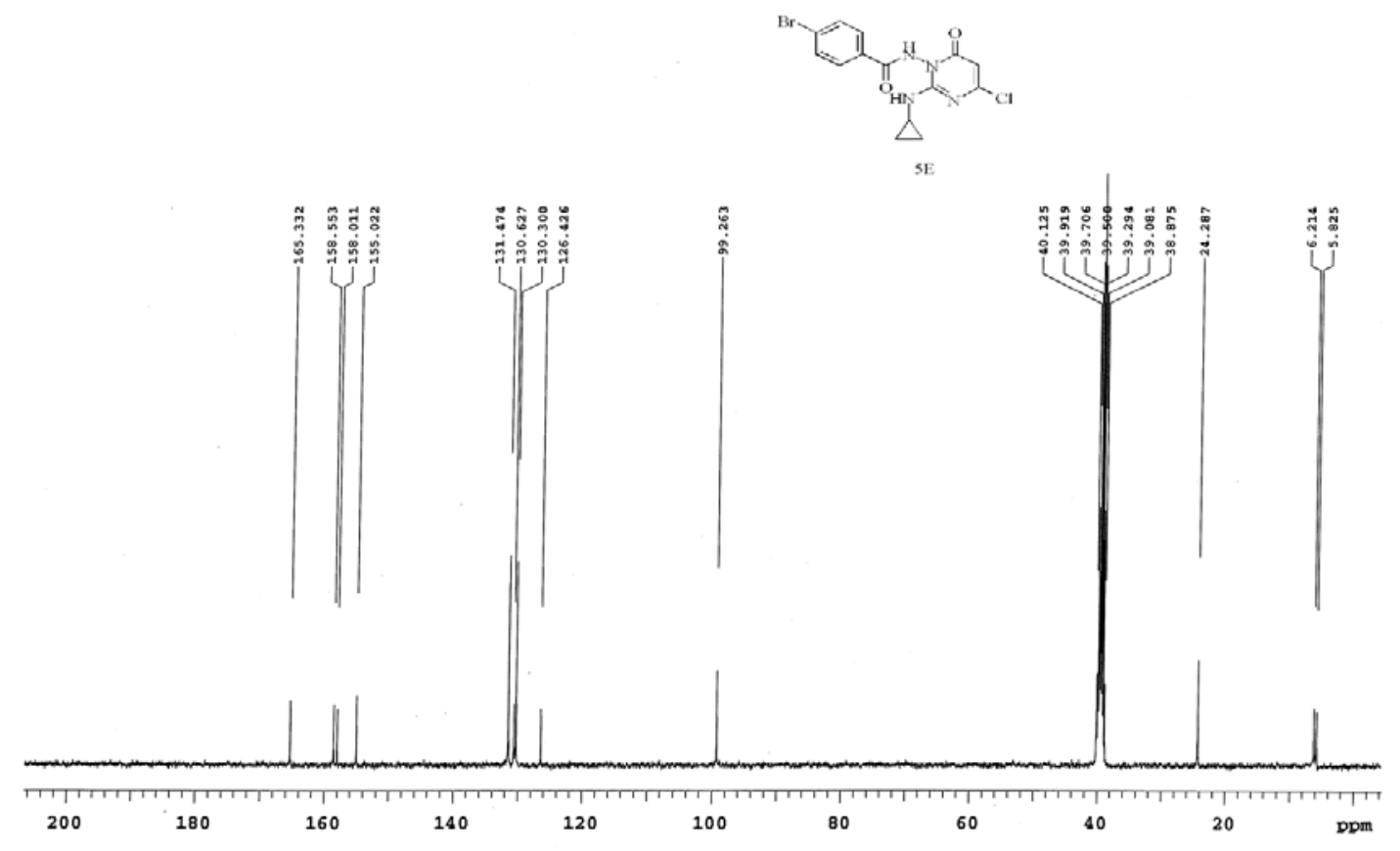

Figure S16. ${ }^{13} \mathrm{C}$ NMR Spectrum of 5e (75 MHz, DMSO-d6, $\left.298 \mathrm{~K}\right)$. 


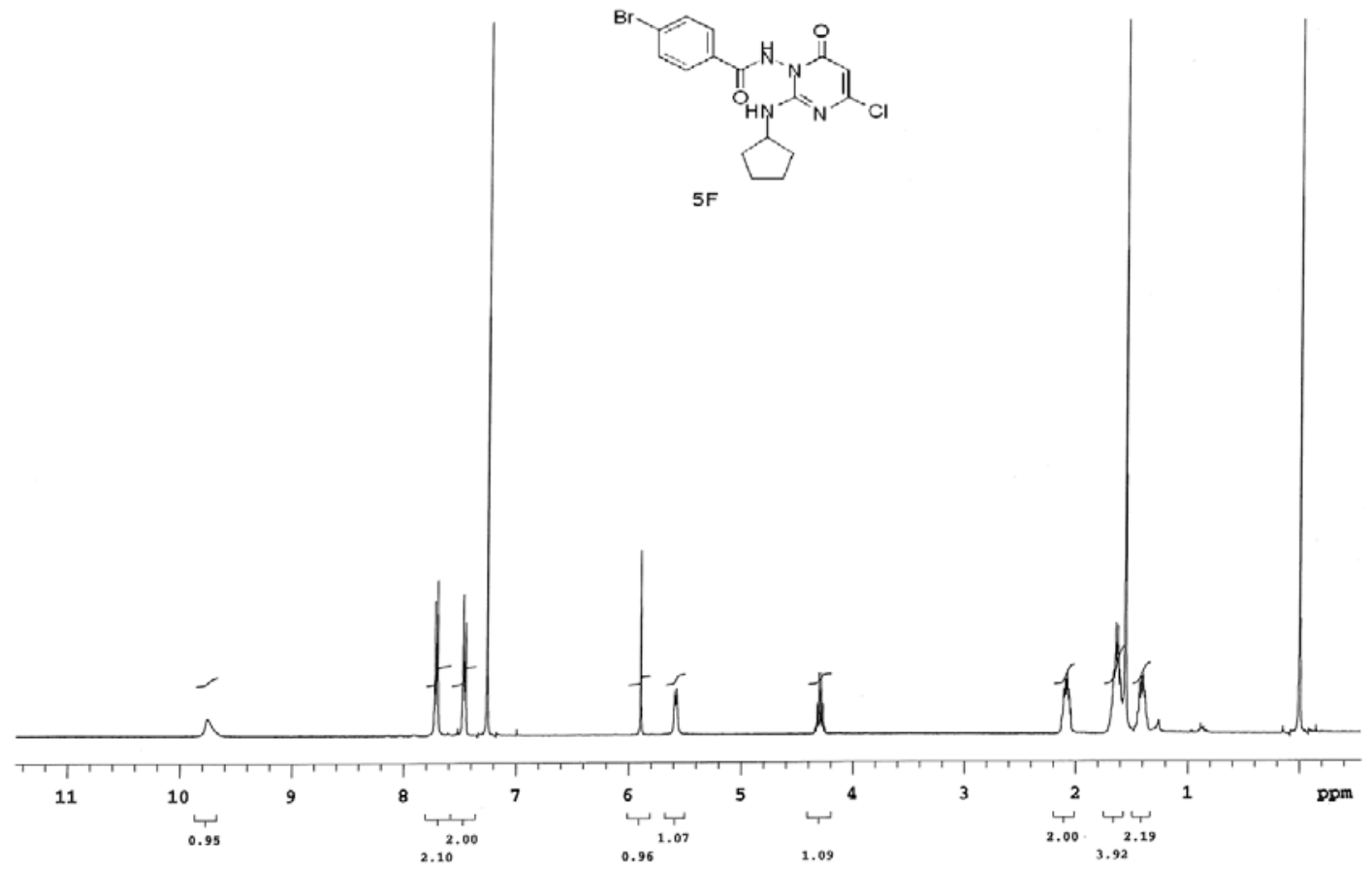

Figure S17. ${ }^{1} \mathrm{H}$ NMR Spectrum of $\mathbf{5 f}\left(400 \mathrm{MHz}, \mathrm{CDCl}_{3}, 298 \mathrm{~K}\right)$. 


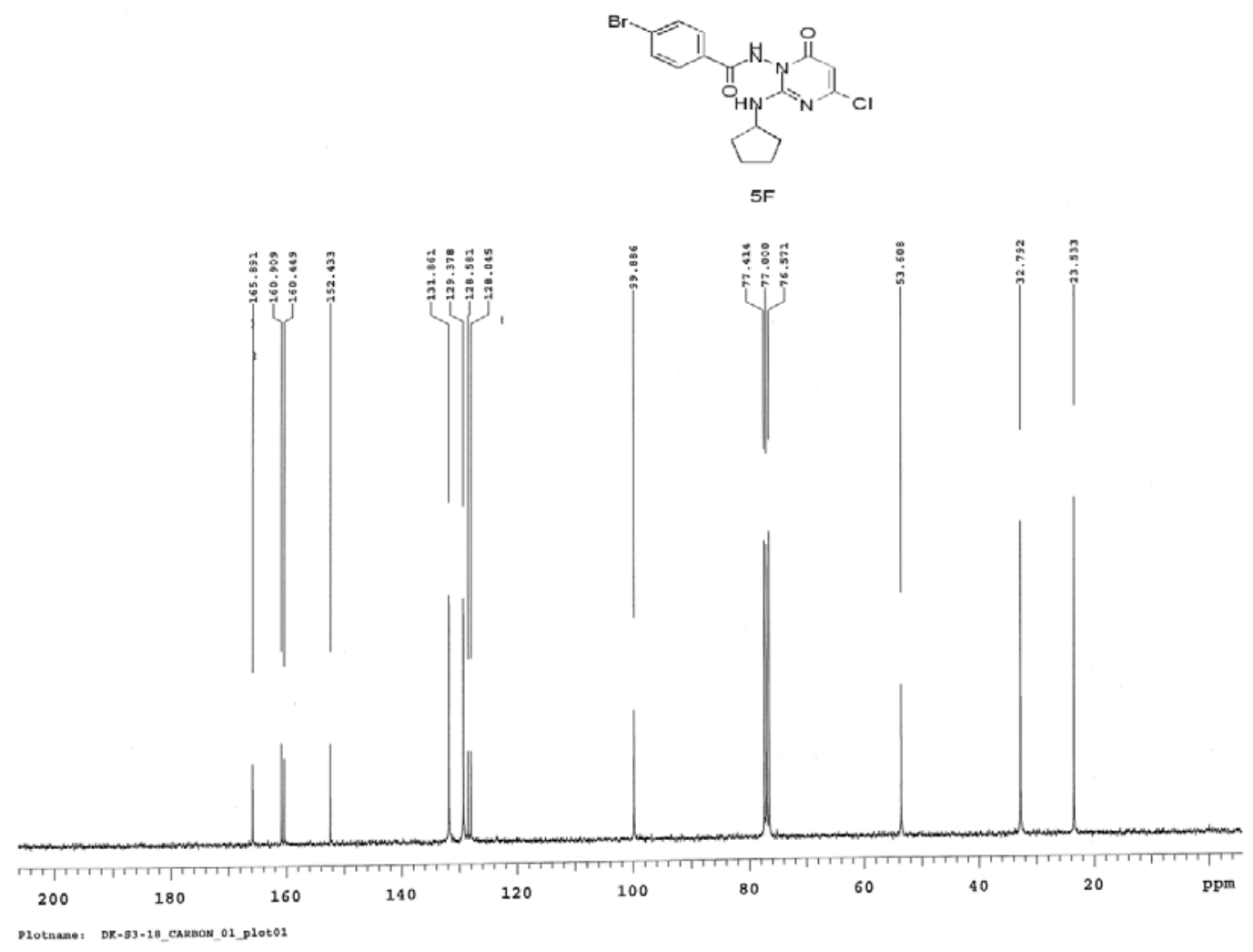

Figure S18. ${ }^{13} \mathrm{C}$ NMR Spectrum of $\mathbf{5 f}\left(75 \mathrm{MHz}, \mathrm{CDCl}_{3}, 298 \mathrm{~K}\right)$. 


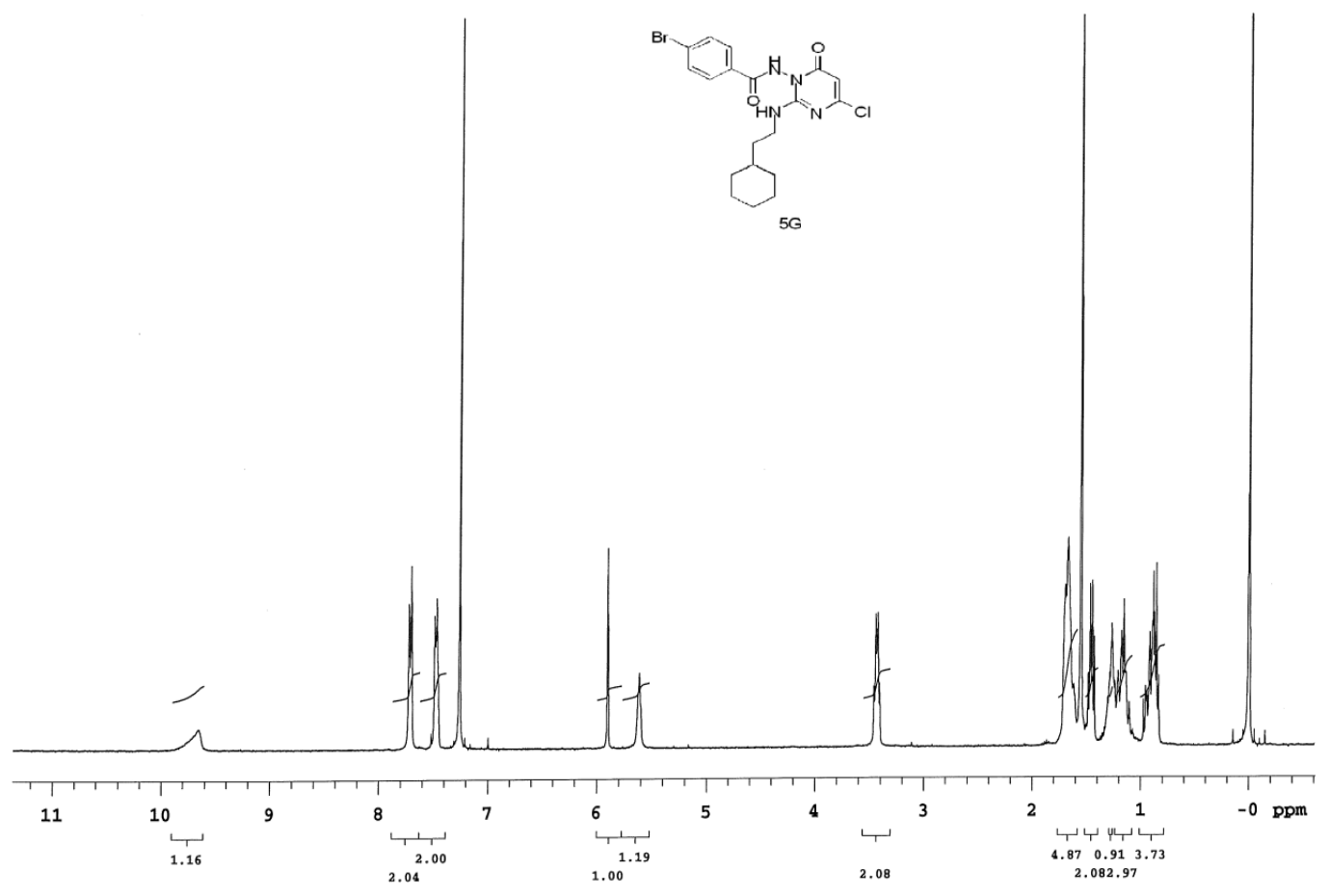

Figure S19. ${ }^{1} \mathrm{H} \mathrm{NMR}$ Spectrum of $\mathbf{5 g}\left(400 \mathrm{MHz}, \mathrm{CDCl}_{3}, 298 \mathrm{~K}\right)$. 


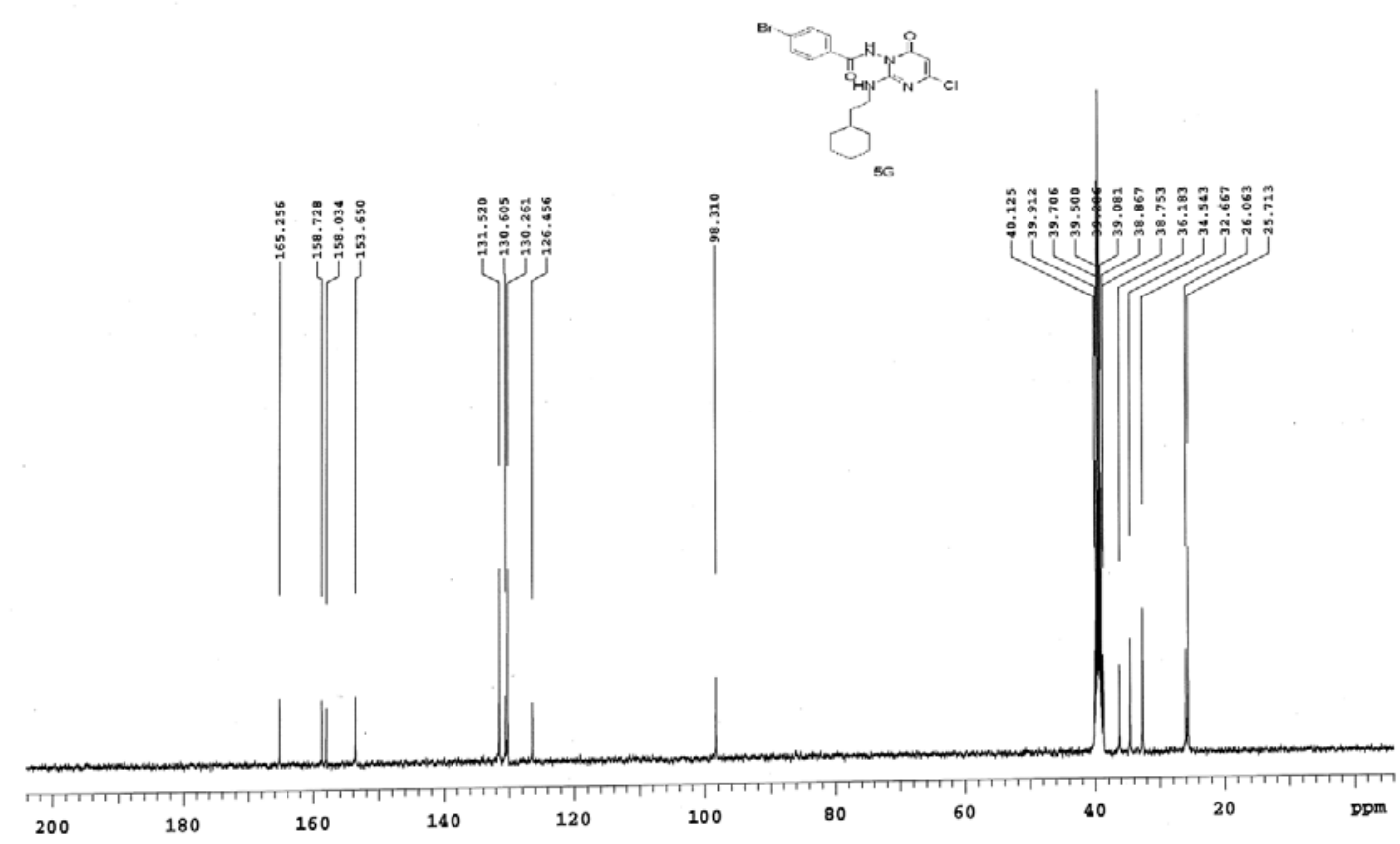

Figure S20. ${ }^{13} \mathrm{C}$ NMR Spectrum of $\mathbf{5 G}(75 \mathrm{MHz}, \mathrm{DMSO}-d 6,298 \mathrm{~K})$. 


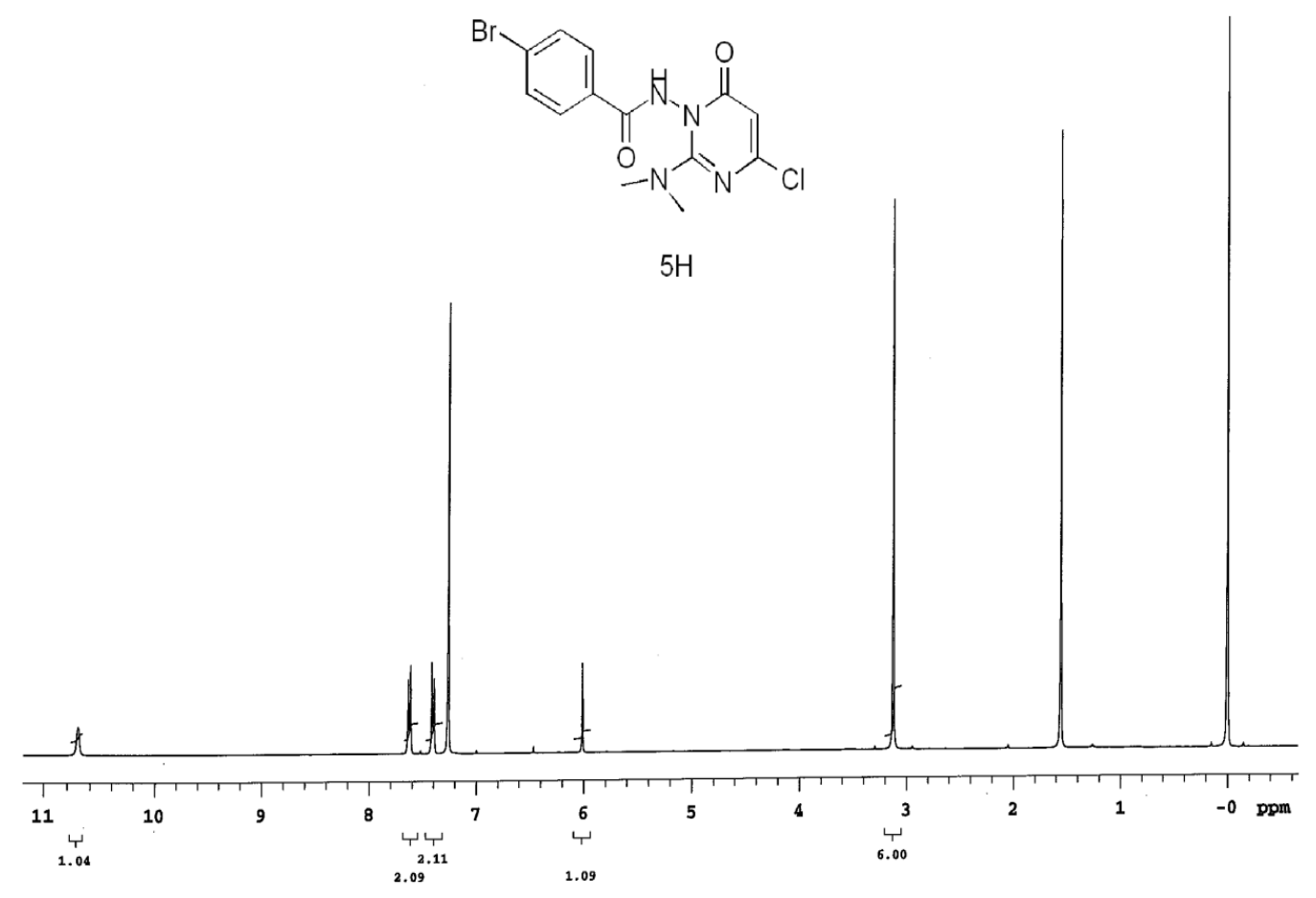

Figure S21. ${ }^{1} \mathrm{H}$ NMR Spectrum of $\mathbf{5 h}\left(400 \mathrm{MHz}, \mathrm{CDCl}_{3}, 298 \mathrm{~K}\right)$. 


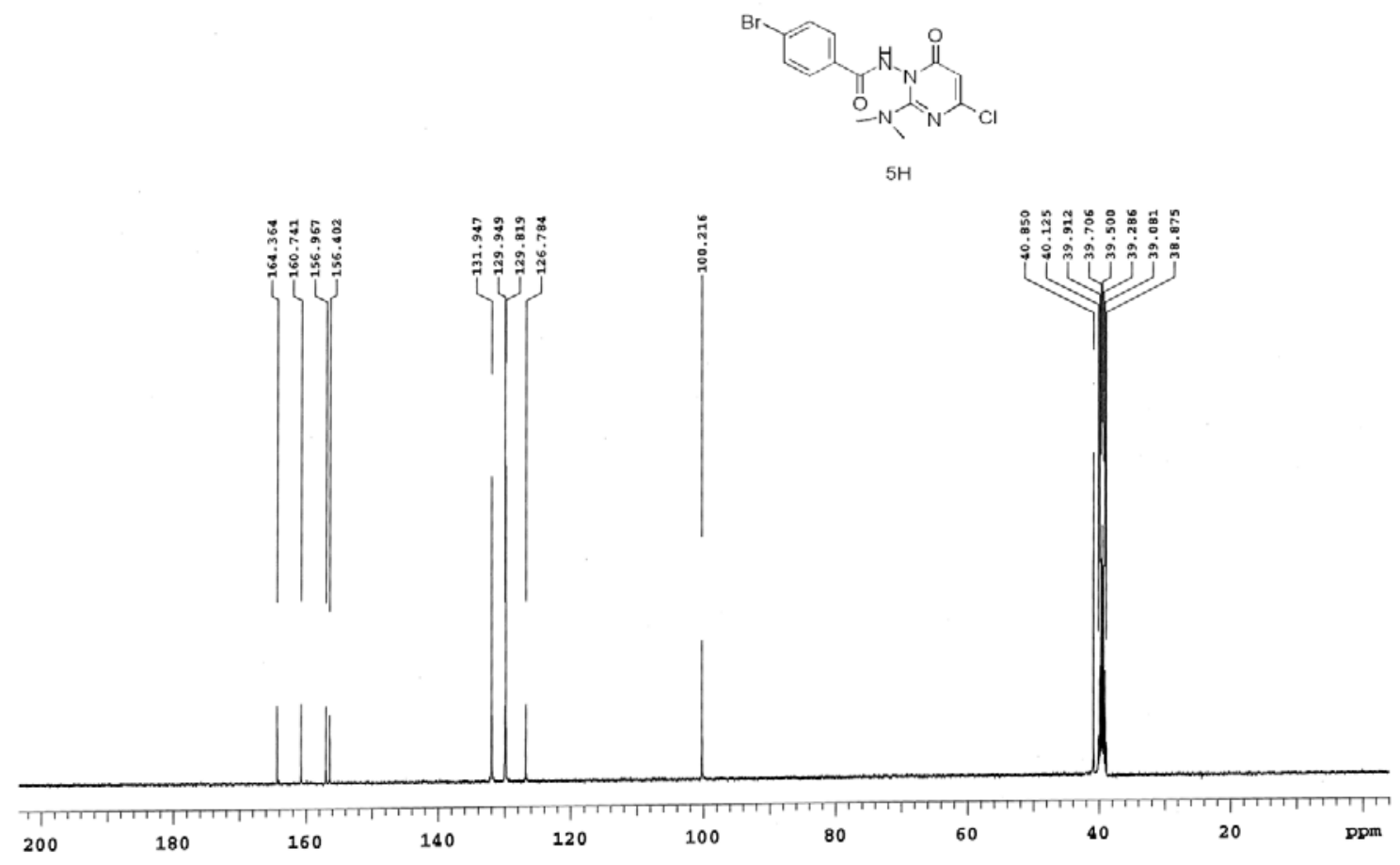

Figure S22. ${ }^{13} \mathrm{C}$ NMR Spectrum of $\mathbf{5 h}(75 \mathrm{MHz}, \mathrm{DMSO}-d 6,298 \mathrm{~K})$. 


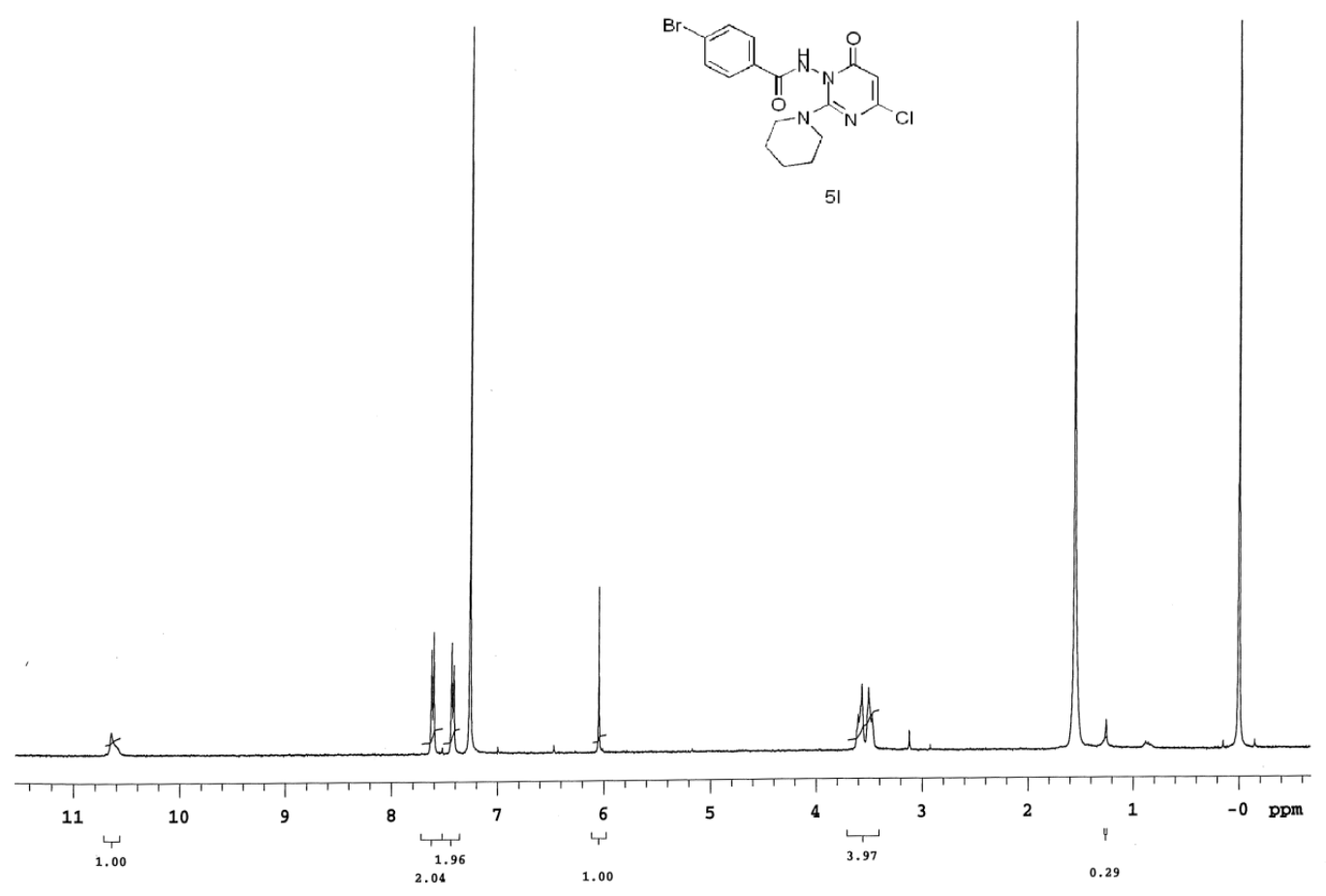

Figure S23. ${ }^{1} \mathrm{H}$ NMR Spectrum of $5 \mathbf{i}\left(400 \mathrm{MHz}, \mathrm{CDCl}_{3}, 298 \mathrm{~K}\right)$. 


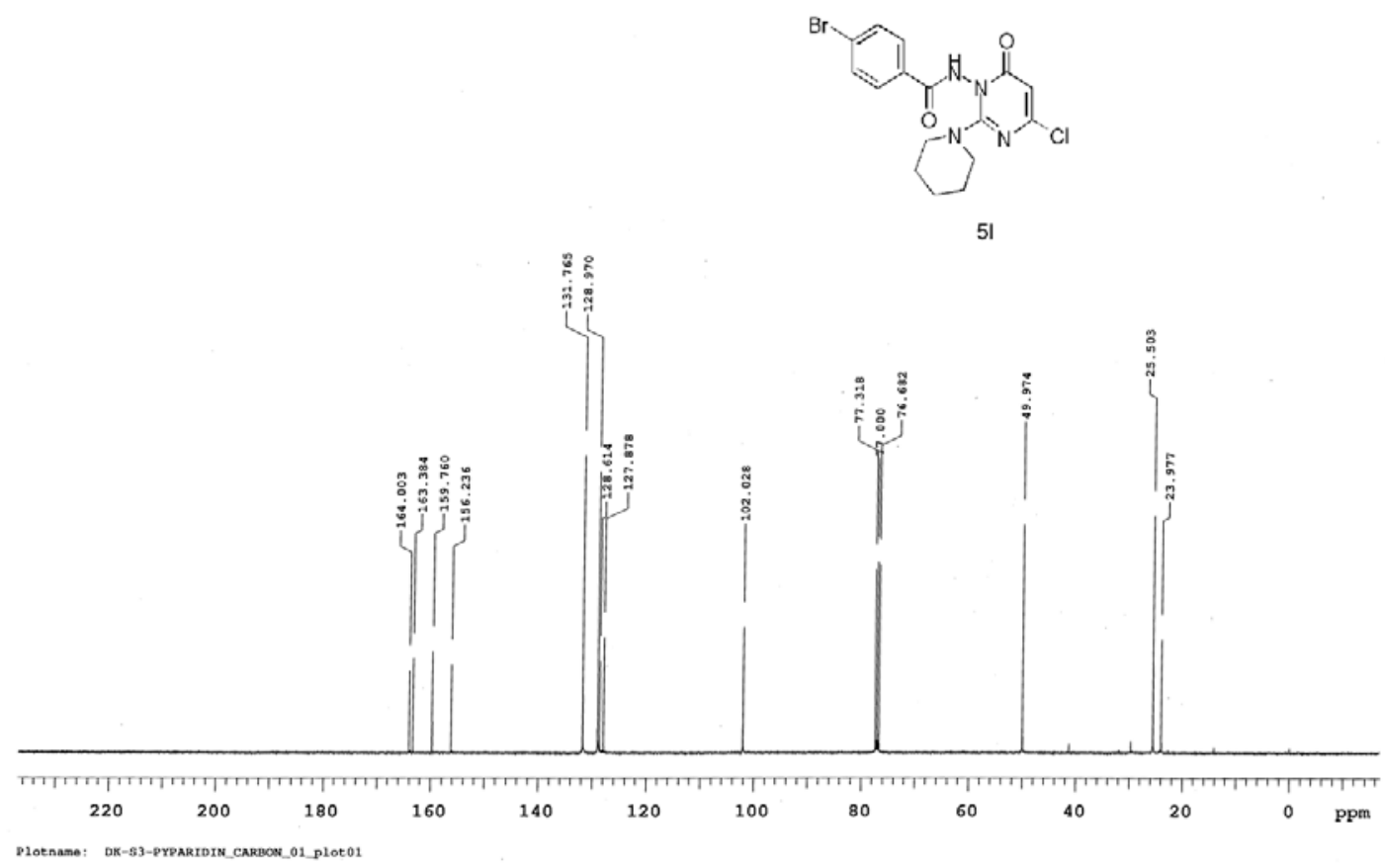

Figure S24. ${ }^{13} \mathrm{C}$ NMR Spectrum of $5 \mathbf{i}\left(75 \mathrm{MHz}, \mathrm{CDCl}_{3}, 298 \mathrm{~K}\right)$. 


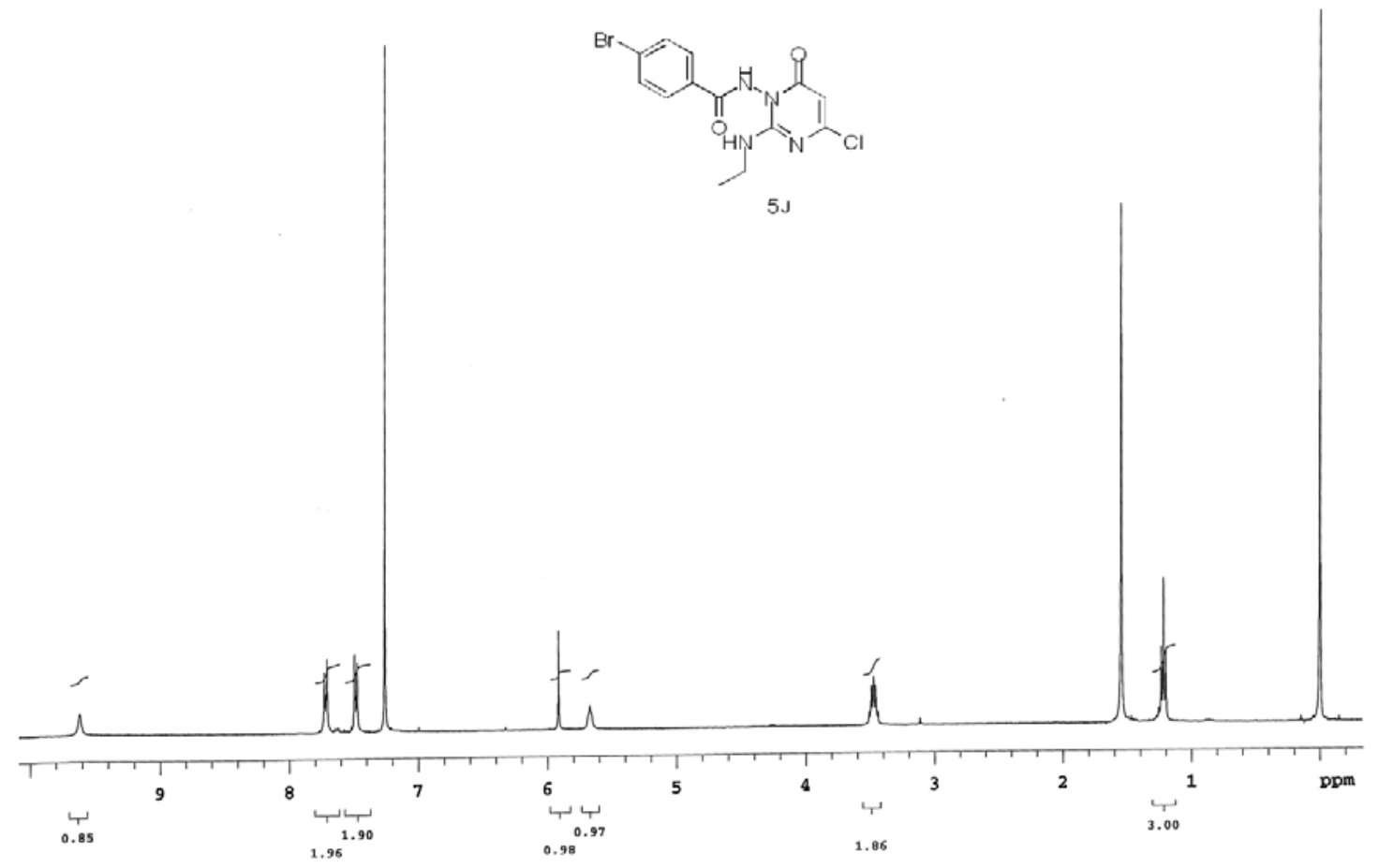

Figure S25. ${ }^{1} \mathrm{H}$ NMR Spectrum of $5 \mathbf{i}\left(400 \mathrm{MHz}, \mathrm{CDCl}_{3}, 298 \mathrm{~K}\right)$. 


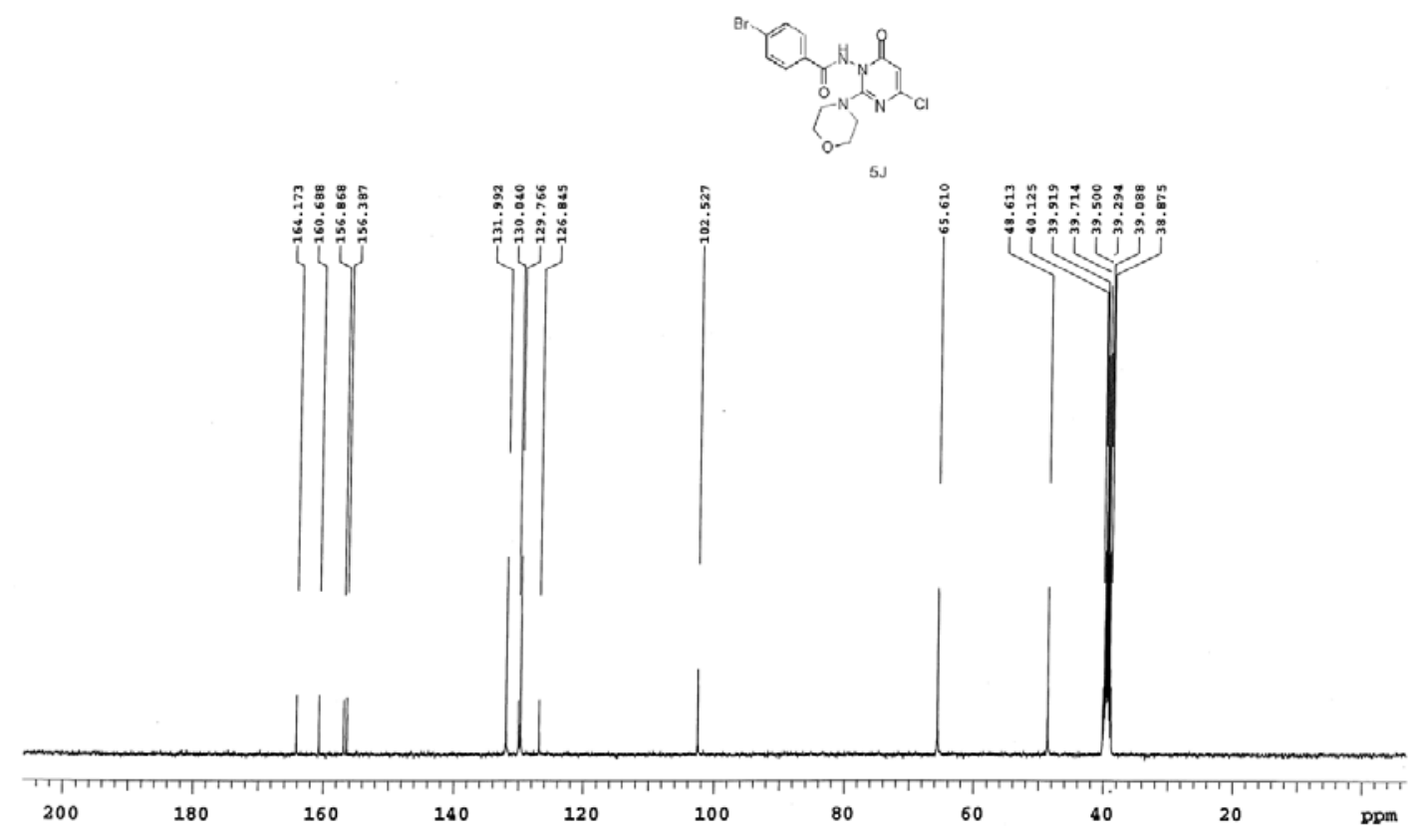

Figure S26. ${ }^{13} \mathrm{C}$ NMR Spectrum of 5j (75 MHz, DMSO-d6, $298 \mathrm{~K}$ ). 


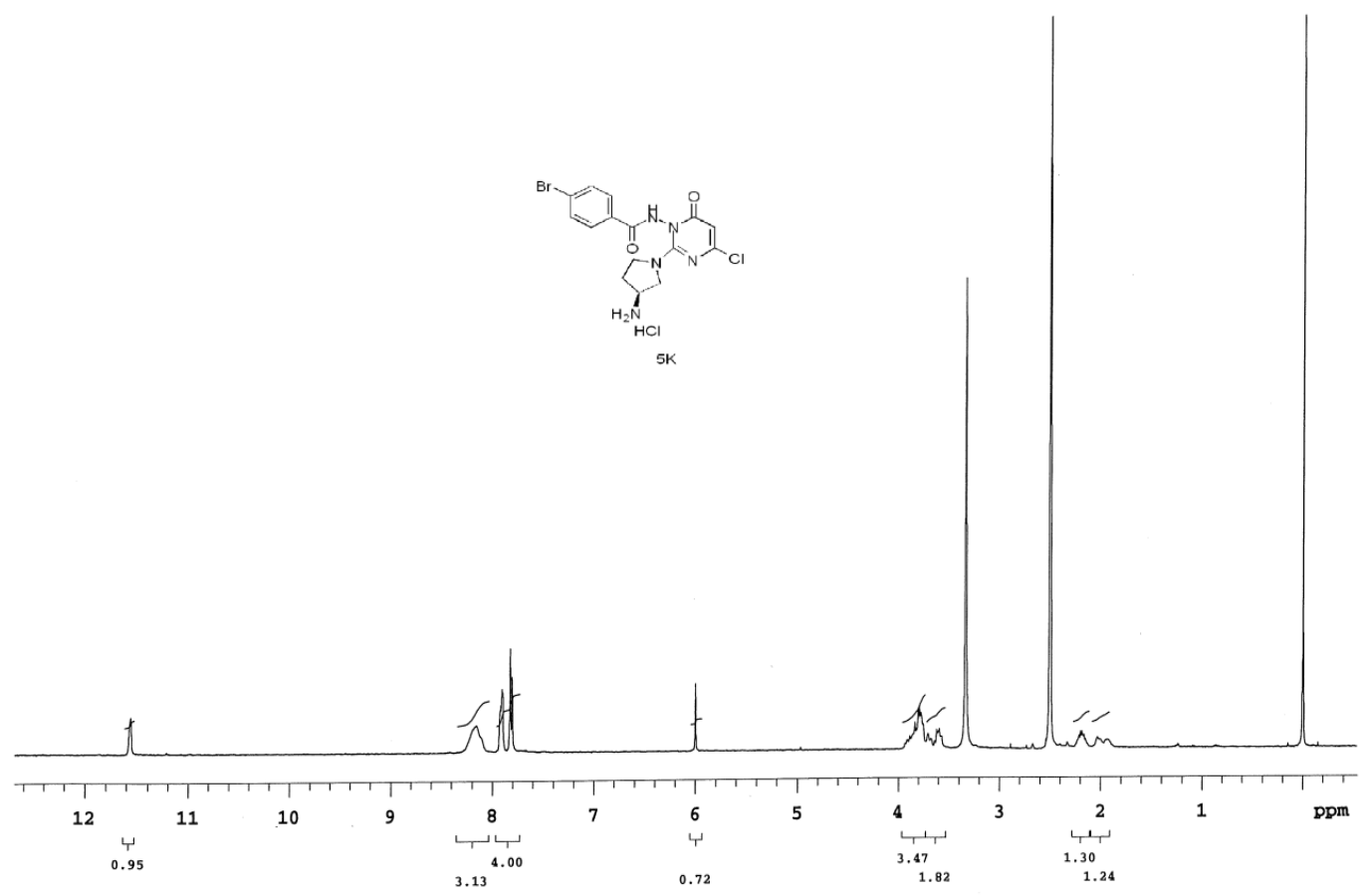

Figure S27. ${ }^{1} \mathrm{H}$ NMR Spectrum of $\mathbf{5 k}\left(400 \mathrm{MHz}, \mathrm{CDCl}_{3}, 298 \mathrm{~K}\right)$. 


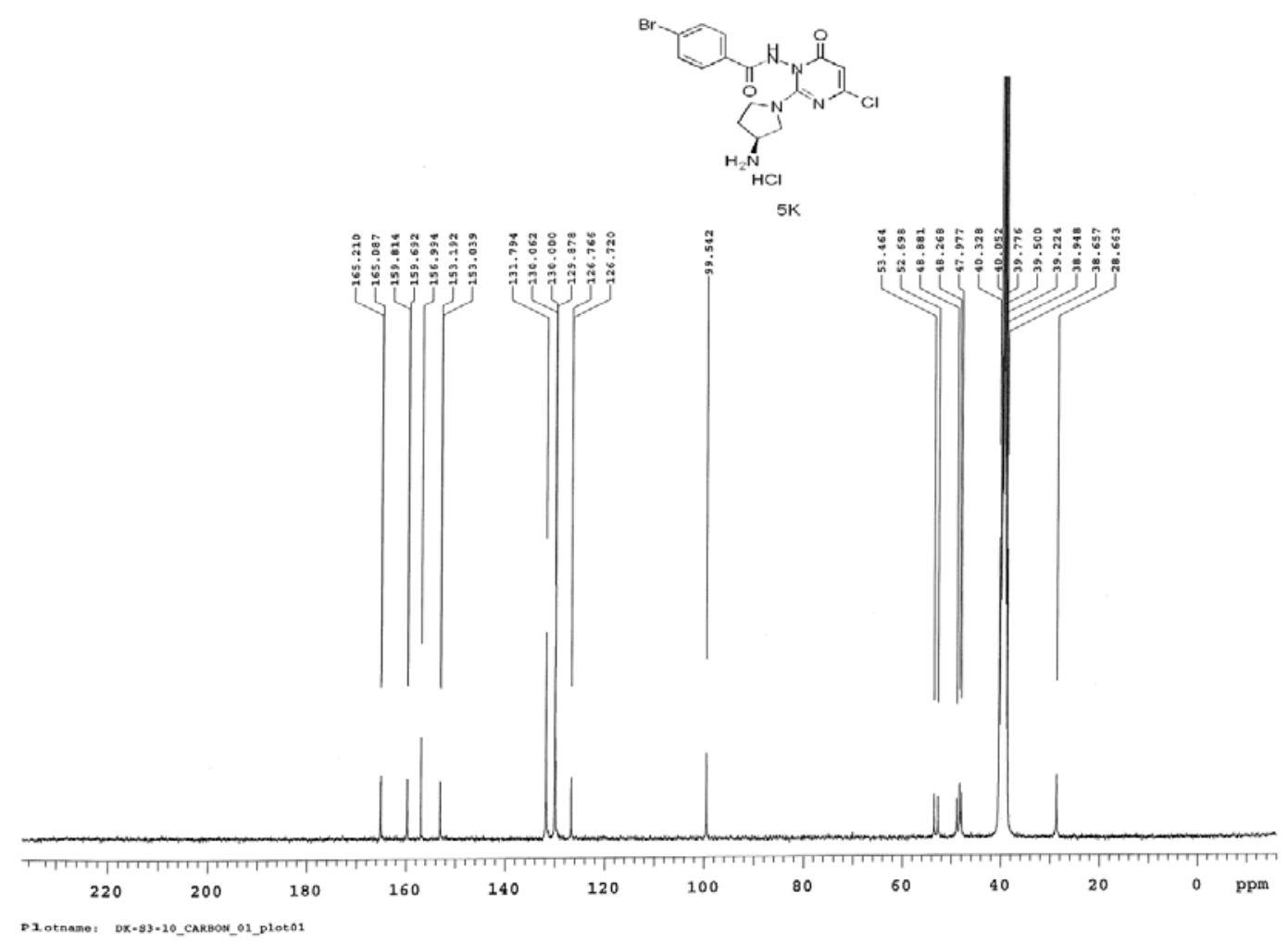

Figure S28. ${ }^{13} \mathrm{C}$ NMR Spectrum of 5k (75 MHz, DMSO-d6, $298 \mathrm{~K}$ ). 


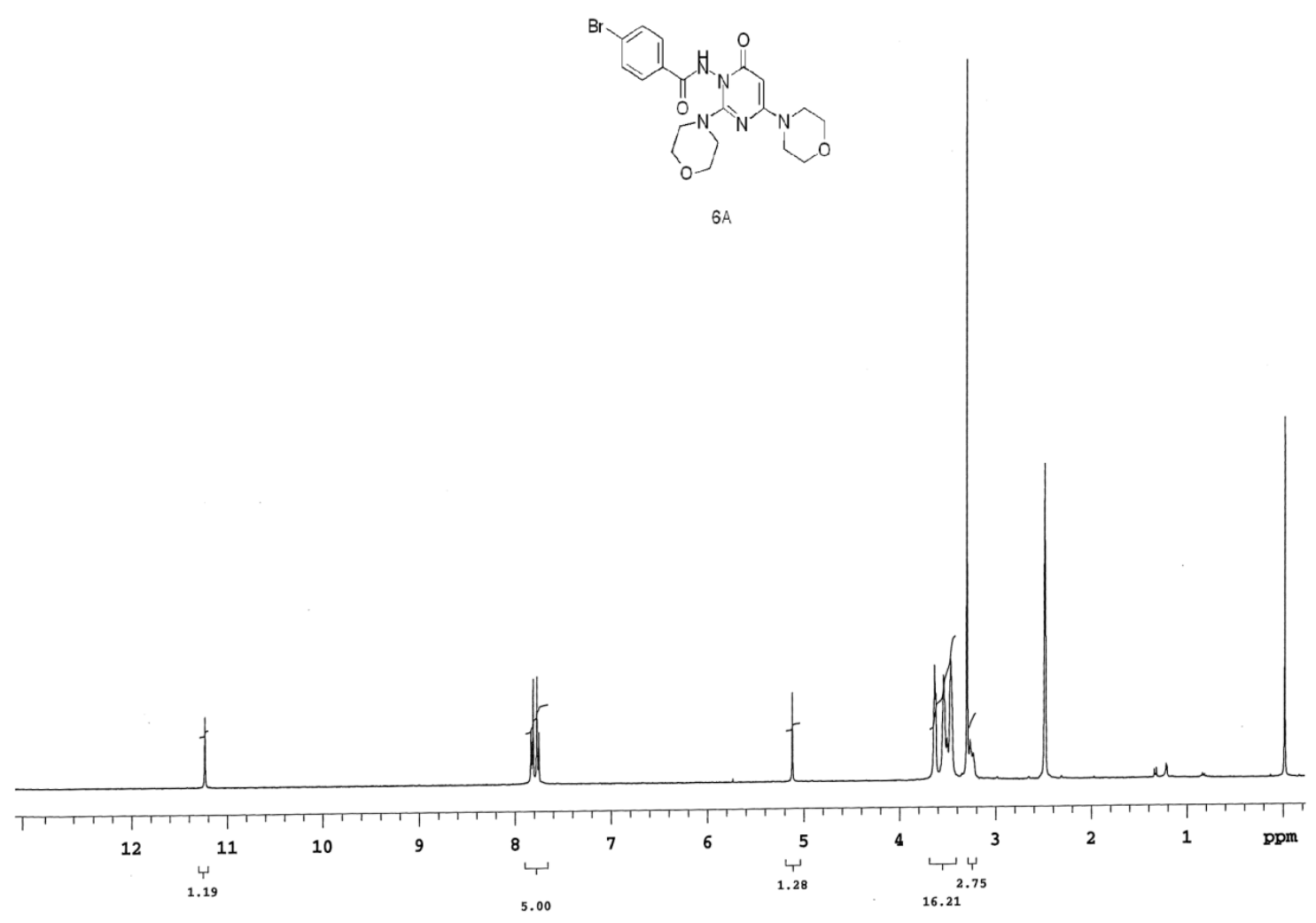

Figure S29. ${ }^{1} \mathrm{H} \mathrm{NMR}$ Spectrum of $6 \mathbf{a}\left(300 \mathrm{MHz}, \mathrm{CDCl}_{3}, 298 \mathrm{~K}\right)$. 


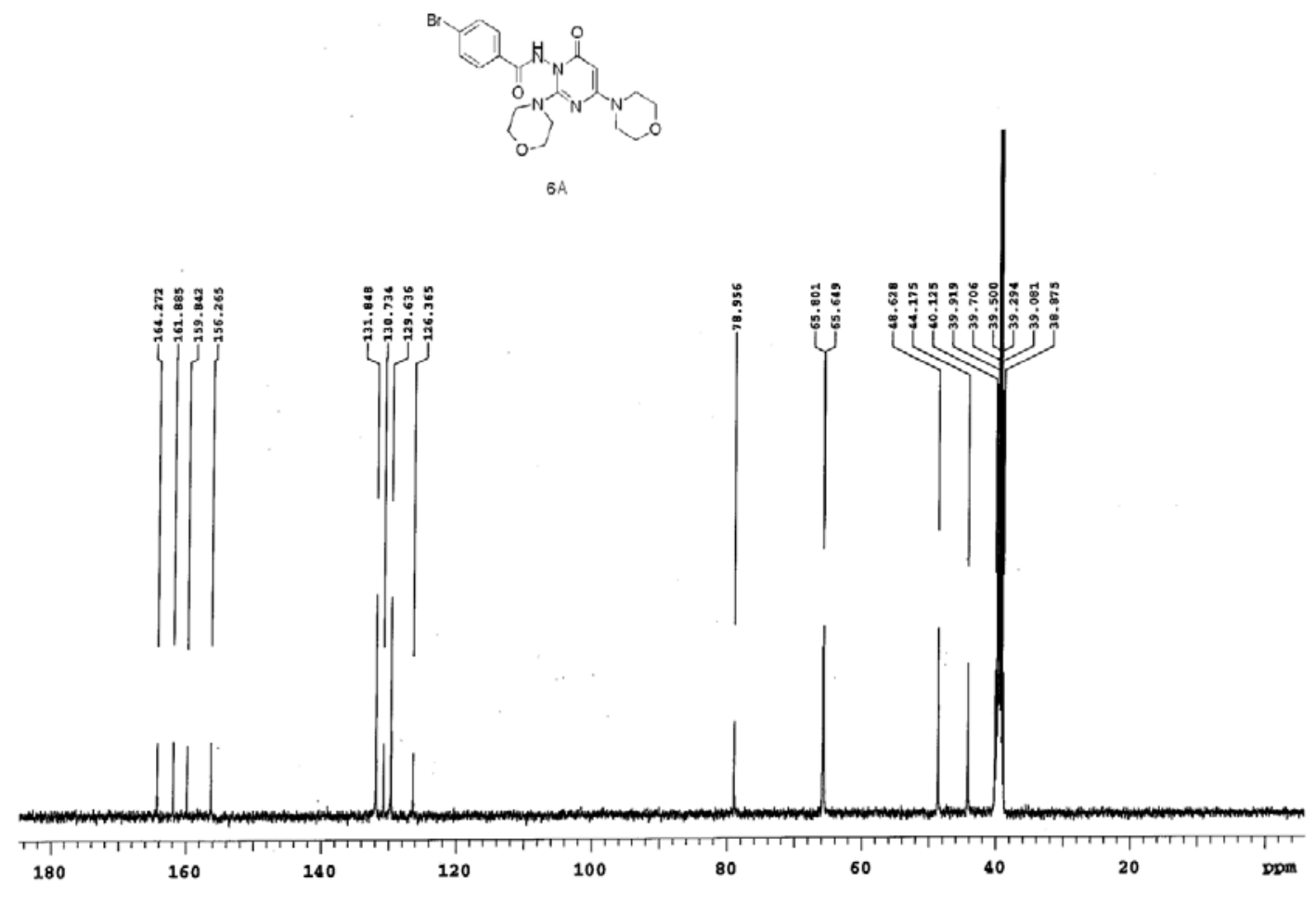

Figure S30. ${ }^{13} \mathrm{C}$ NMR Spectrum of $6 \mathbf{a}\left(75 \mathrm{MHz}, \mathrm{CDCl}_{3}, 298 \mathrm{~K}\right)$. 


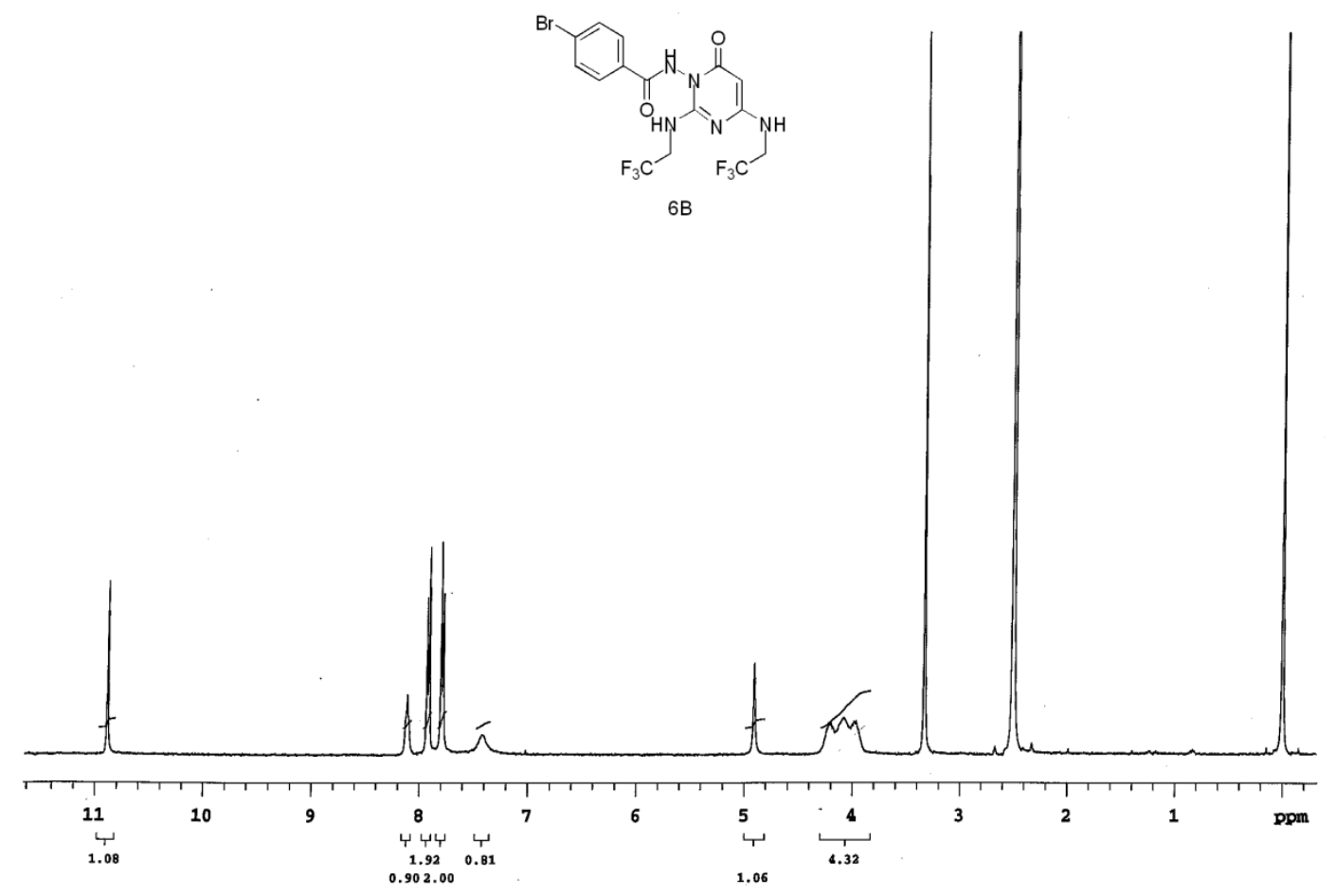

Figure S31. ${ }^{1} \mathrm{H}$ NMR Spectrum of $6 \mathbf{b}(400 \mathrm{MHz}$, DMSO-d6, $298 \mathrm{~K})$. 


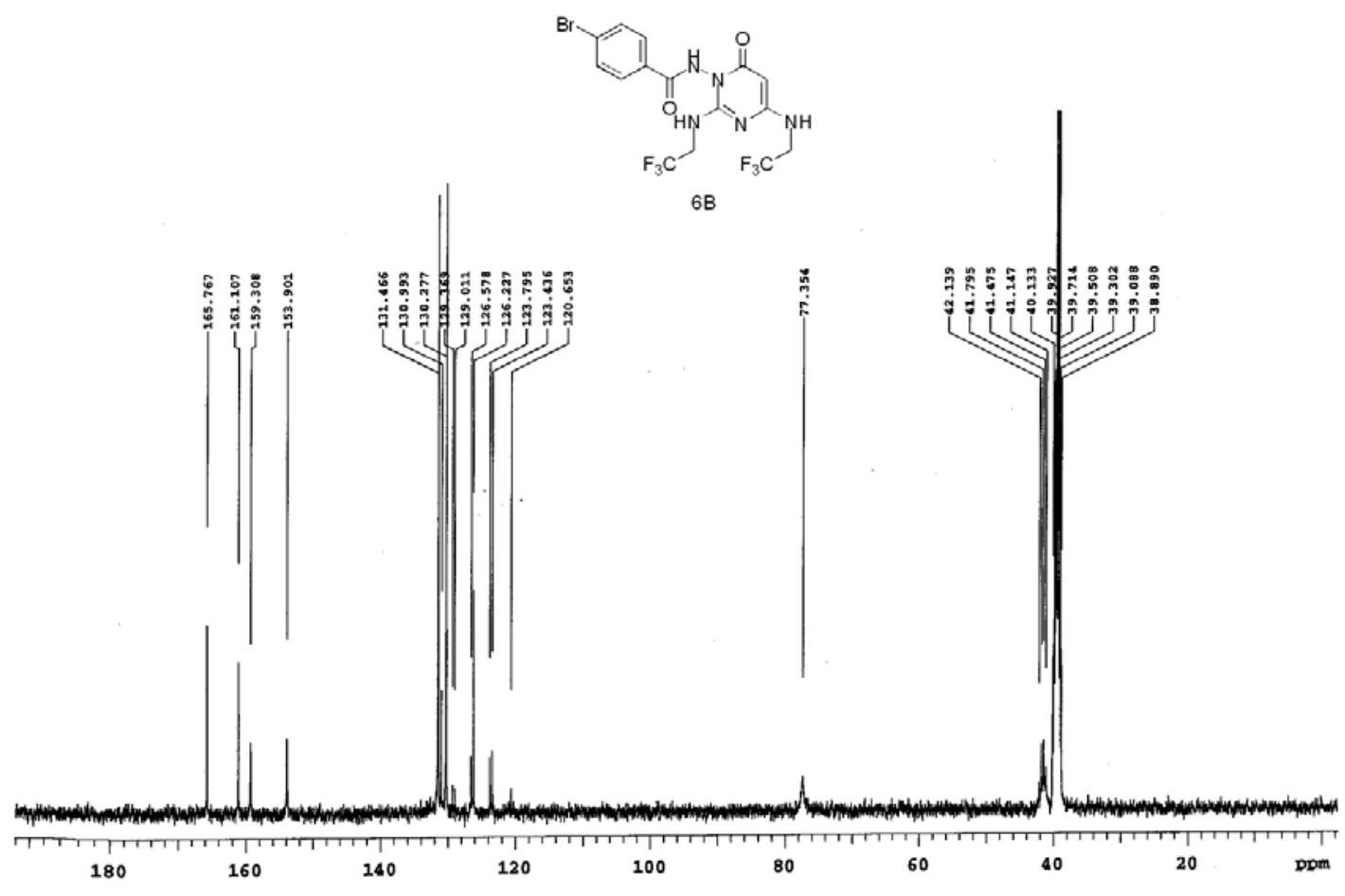

Figure S32. ${ }^{13} \mathrm{C}$ NMR Spectrum of $6 \mathbf{b}(75 \mathrm{MHz}, \mathrm{DMSO}-d 6,298 \mathrm{~K})$. 


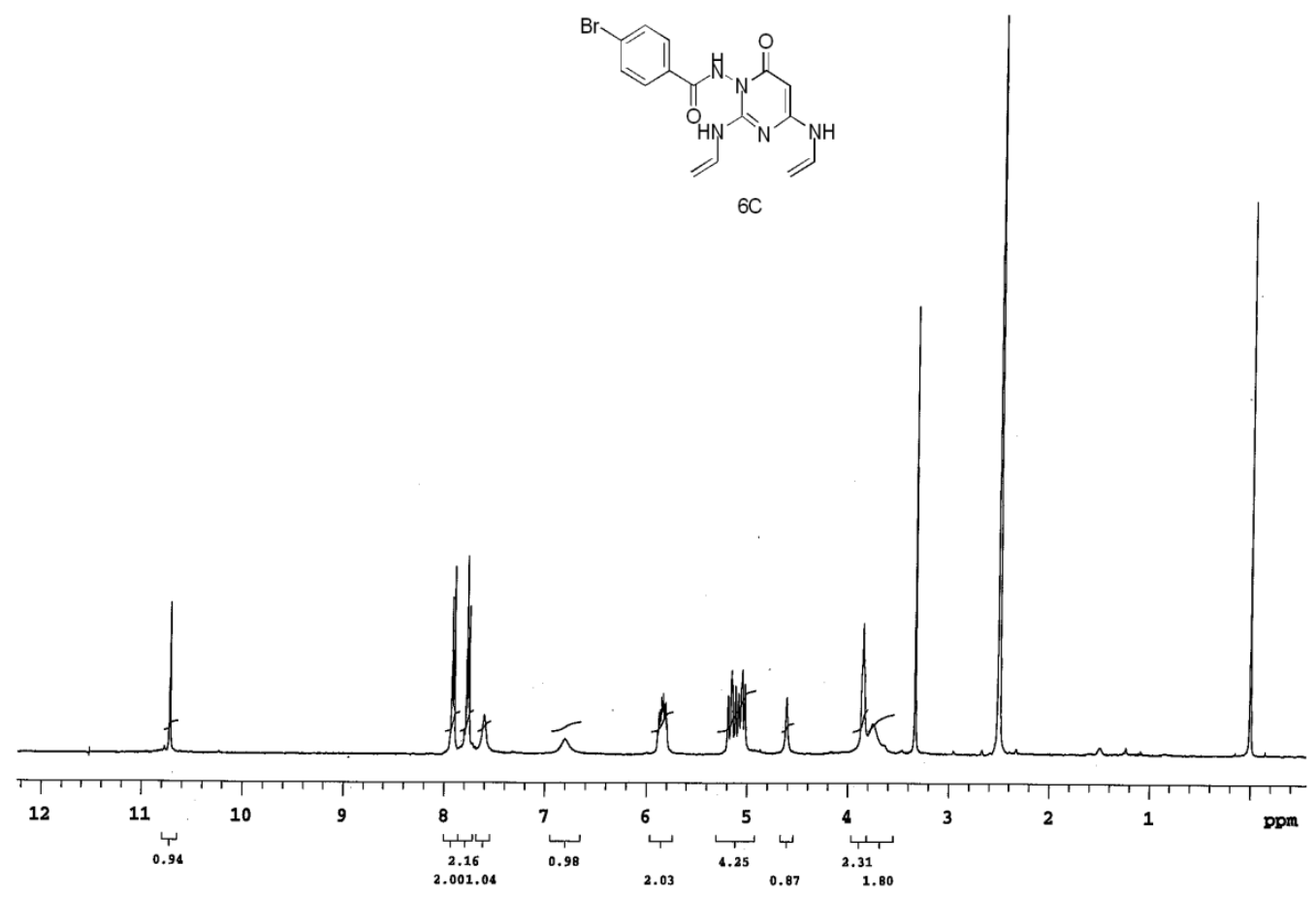

Figure S33. ${ }^{1} \mathrm{H}$ NMR Spectrum of 6c (400 MHz, DMSO-d6, $298 \mathrm{~K}$ ). 


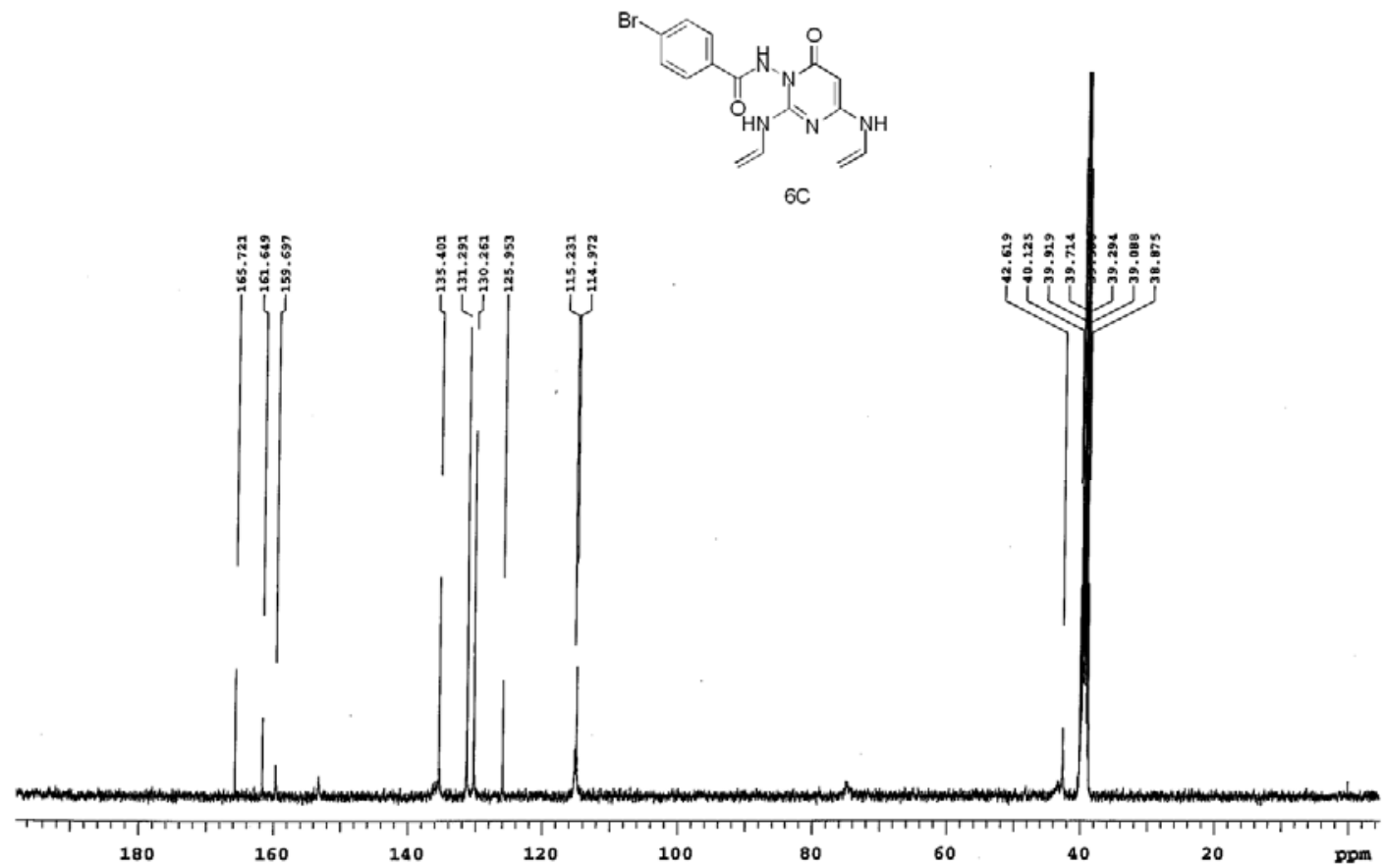

Figure S34. ${ }^{13} \mathrm{C}$ NMR Spectrum of $6 \mathbf{c}(75 \mathrm{MHz}, \mathrm{DMSO}-d 6,298 \mathrm{~K})$. 


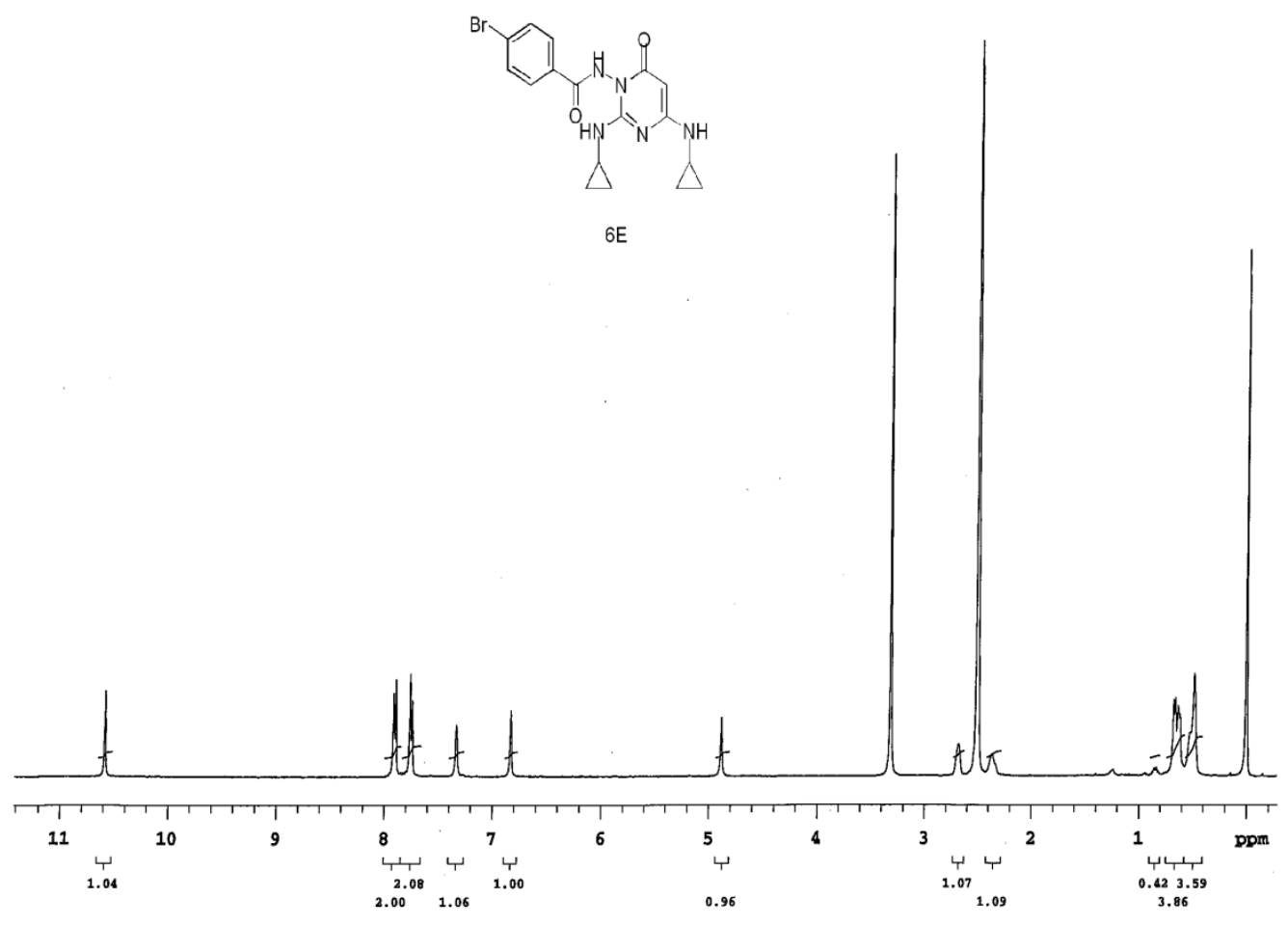

Figure S35. ${ }^{1} \mathrm{H}$ NMR Spectrum of 6e (400 MHz, DMSO-d6, $298 \mathrm{~K}$ ). 


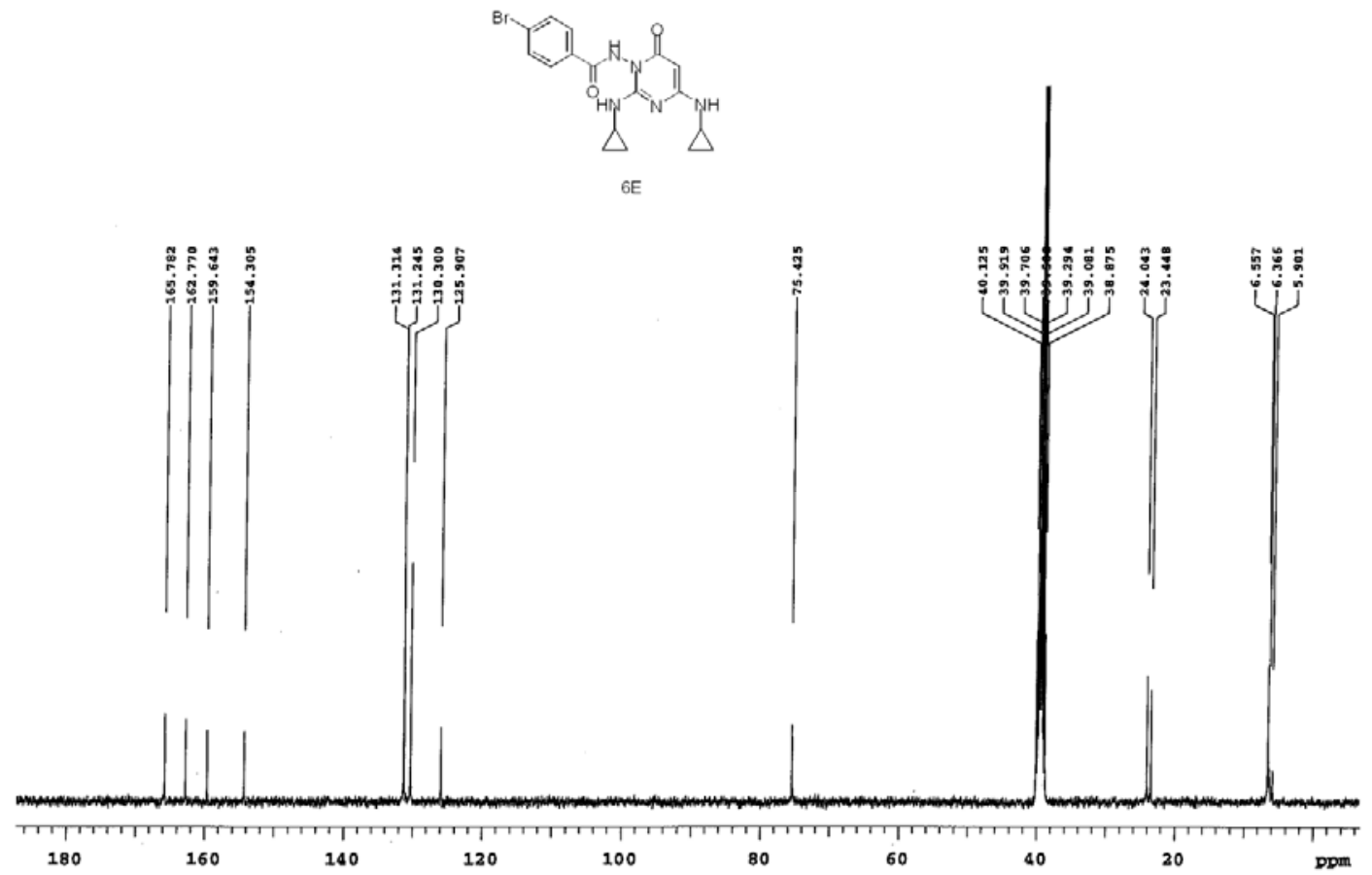

Figure S36. ${ }^{13} \mathrm{C}$ NMR Spectrum of $6 \mathbf{e}(75 \mathrm{MHz}, \mathrm{DMSO}-d 6,298 \mathrm{~K})$. 


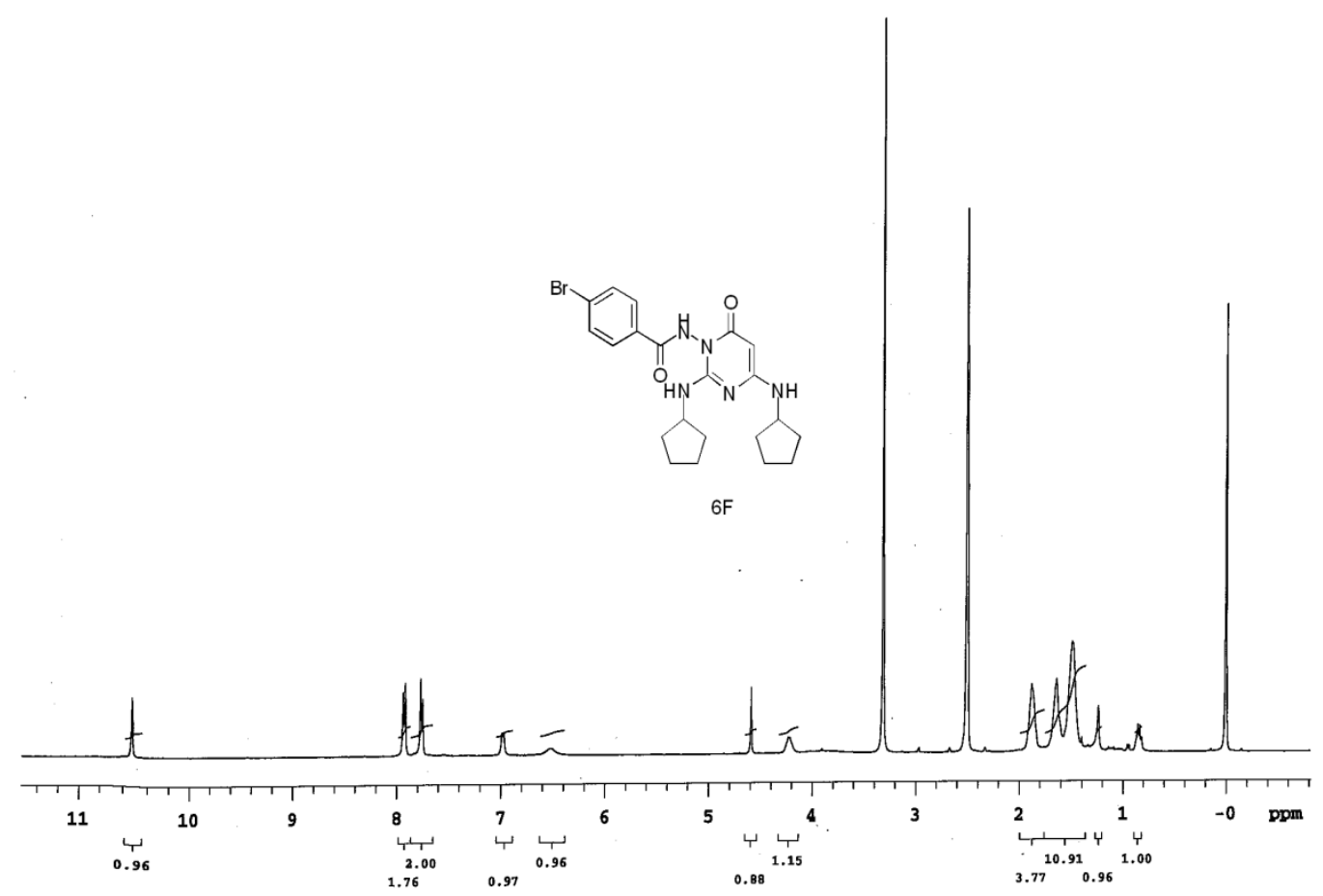

Figure S37. ${ }^{1} \mathrm{H}$ NMR Spectrum of $6 \mathbf{f}(400 \mathrm{MHz}, \mathrm{DMSO}-d 6,298 \mathrm{~K})$. 


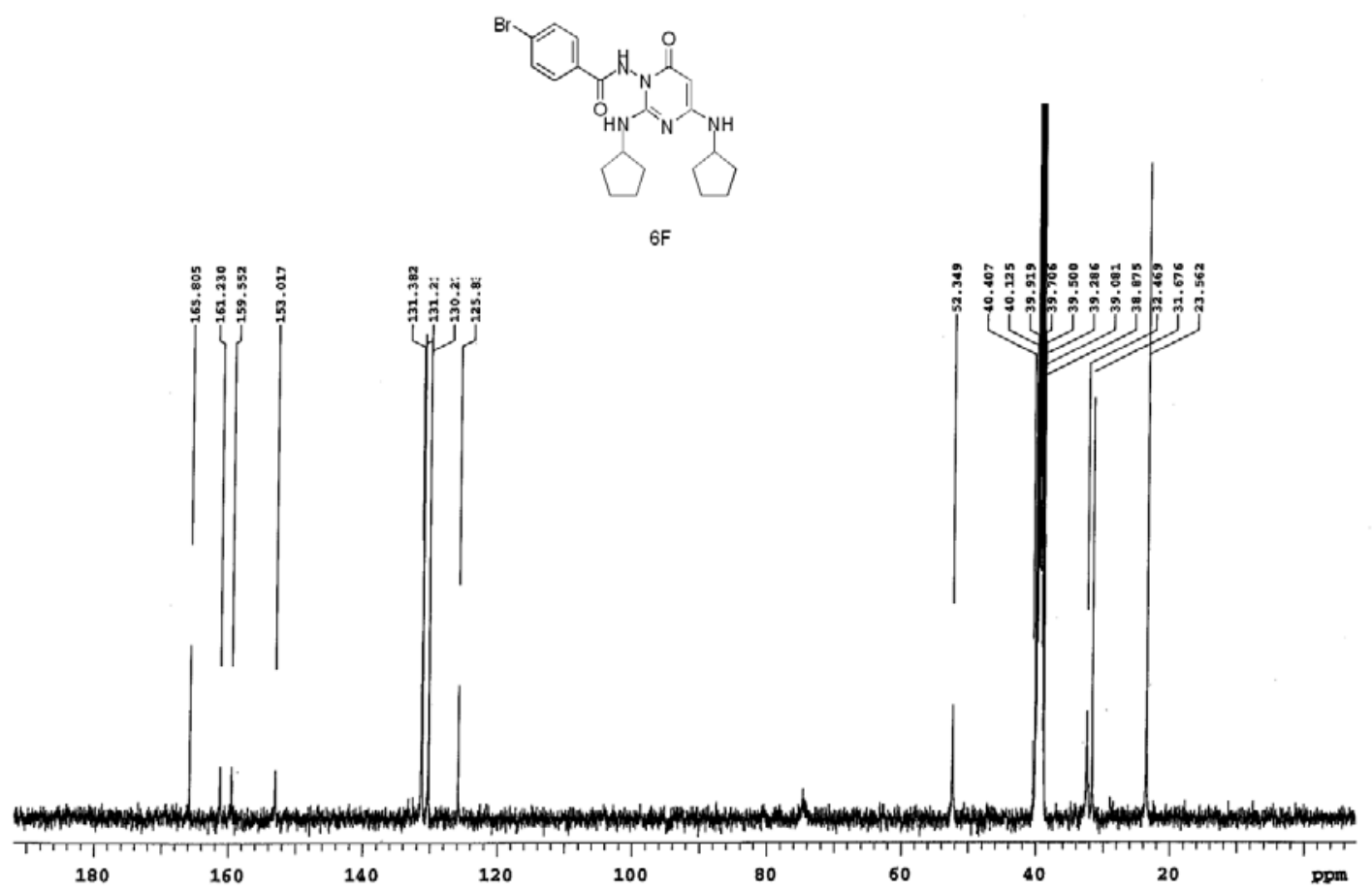

Figure S38. ${ }^{13} \mathrm{C}$ NMR Spectrum of $6 \mathbf{f}(75 \mathrm{MHz}, \mathrm{DMSO}-d 6,298 \mathrm{~K})$. 


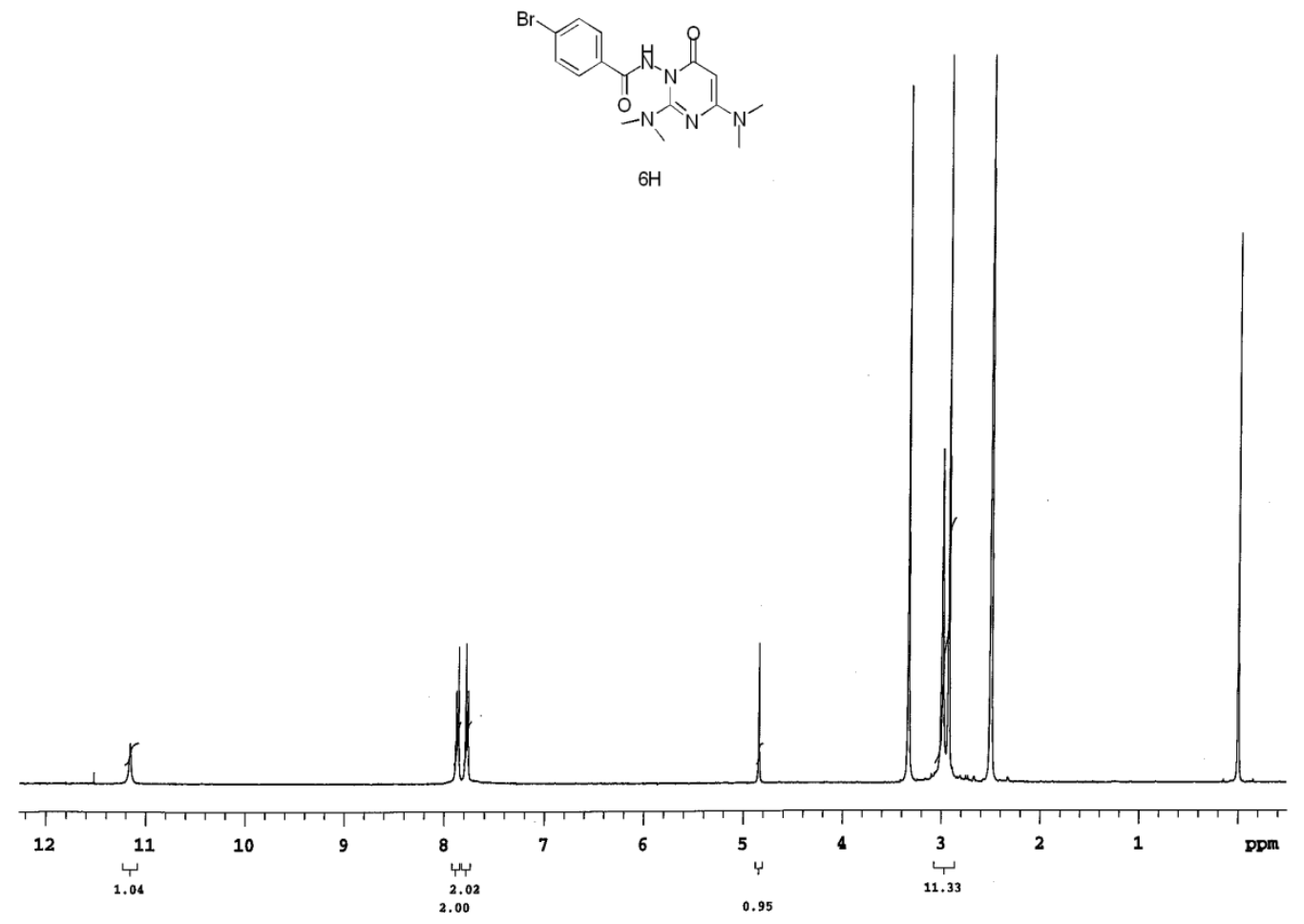

Figure S39. ${ }^{1} \mathrm{H}$ NMR Spectrum of $\mathbf{6 h}(400 \mathrm{MHz}, \mathrm{DMSO}-d 6,298 \mathrm{~K})$. 


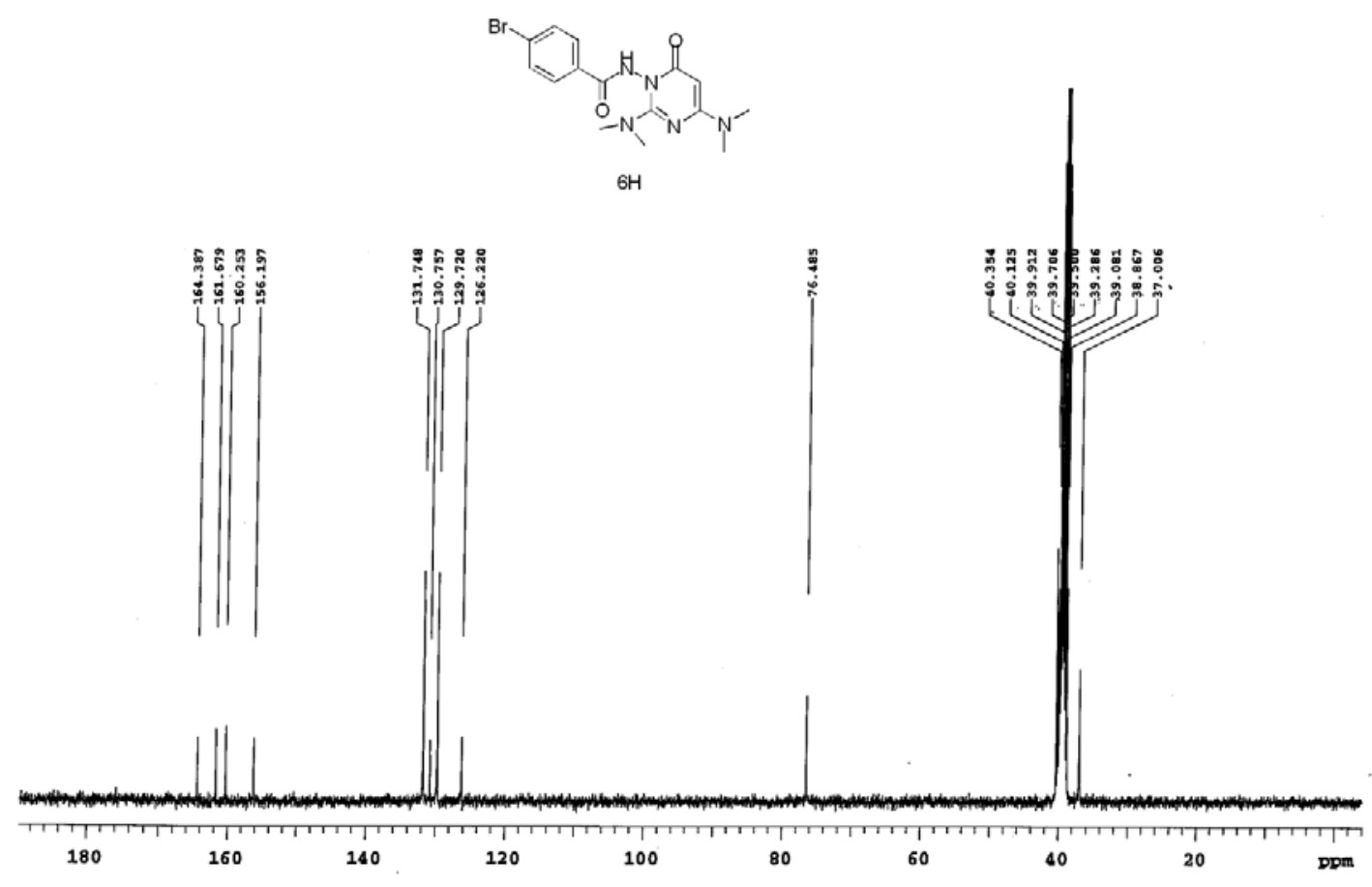

Figure S40. ${ }^{13} \mathrm{C}$ NMR Spectrum of $6 \mathbf{h}(75 \mathrm{MHz}, \mathrm{DMSO}-d 6,298 \mathrm{~K})$. 

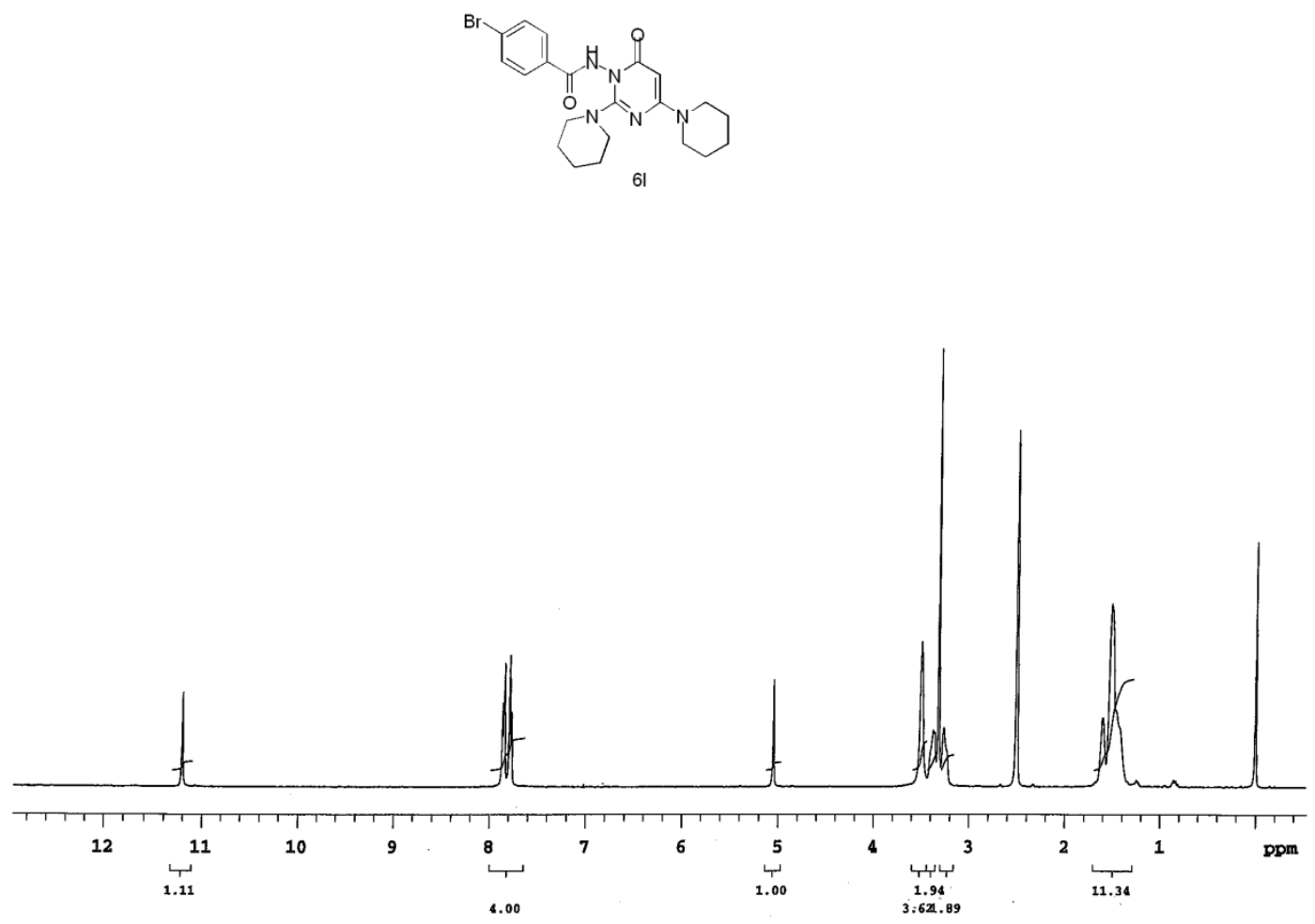

Figure S41. ${ }^{1} \mathrm{H}$ NMR Spectrum of 6i (400 MHz, DMSO-d6, $298 \mathrm{~K}$ ). 

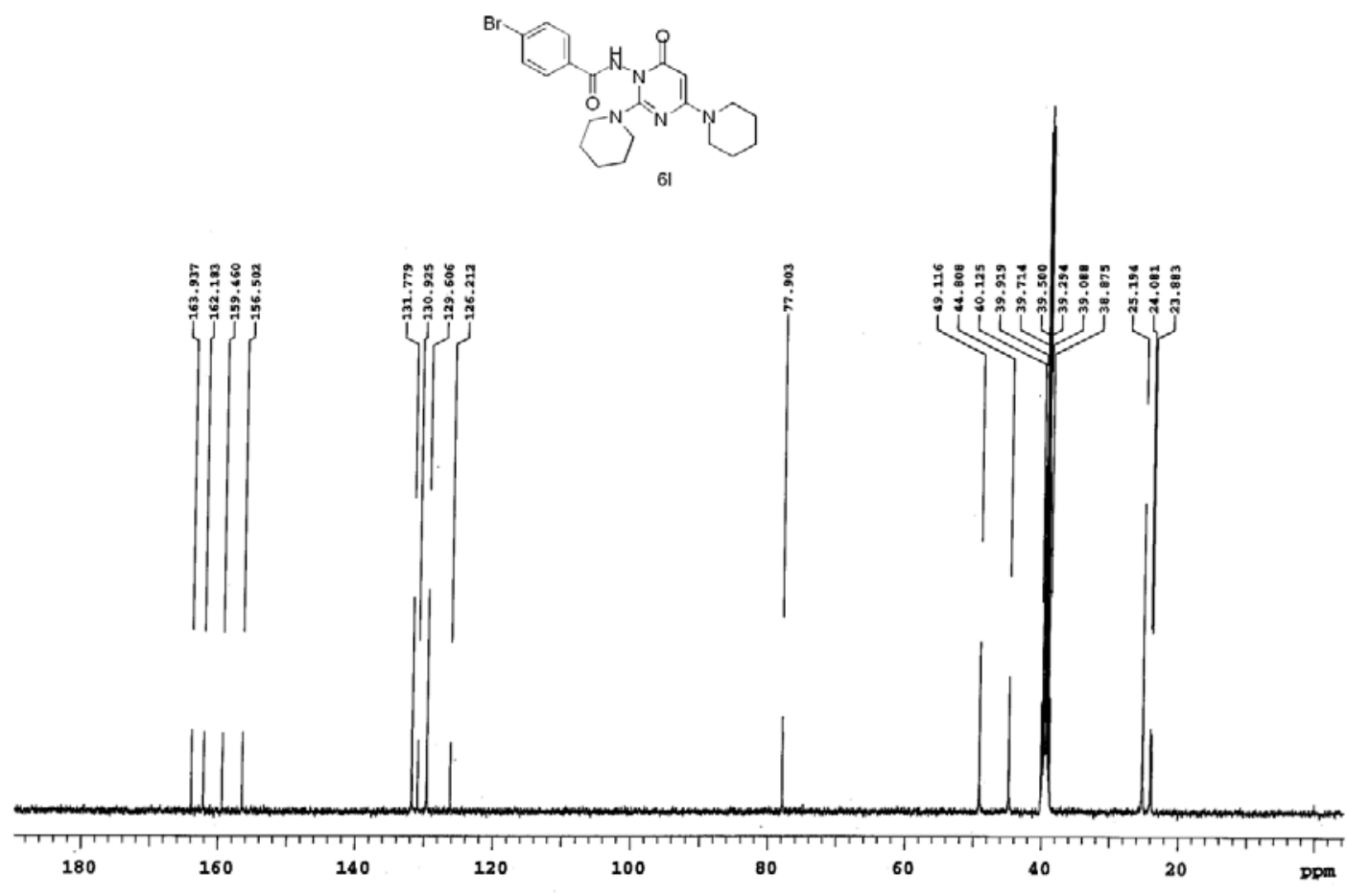

Figure S42. ${ }^{13} \mathrm{C}$ NMR Spectrum of $6 \mathbf{i}(75 \mathrm{MHz}$, DMSO-d6, $298 \mathrm{~K})$. 